\title{
Performance Verification for Robot Missions in Uncertain Environments
}

Damian Lyons

Fordham University, dlyons@fordham.edu

Ron Arkin

Georgia Institute of Technology

Shu Jiang

Matt O'Brien

Feng Tang

See next page for additional authors

Follow this and additional works at: https://fordham.bepress.com/frcv_facultypubs

Part of the Artificial Intelligence and Robotics Commons, and the Robotics Commons

\section{Recommended Citation}

Damian Lyons, Ron Arkin, Shu Jiang, Matthew O'Brien, Feng Tang and Peng Tang, "Performance Verification for Robot Missions in Uncertain Environments" Robotics \& Autonomous Systems 98 (2017) pp89-104.

This Article is brought to you for free and open access by the Robotics and Computer Vision Laboratory at DigitalResearch@Fordham. It has been accepted for inclusion in Faculty Publications by an authorized administrator of DigitalResearch@Fordham. For more information, please contact considine@fordham.edu. 
Authors

Damian Lyons, Ron Arkin, Shu Jiang, Matt O'Brien, Feng Tang, and Peng Tang 


\title{
Performance verification for robot missions in uncertain environments
}

\author{
D.M. Lyons ${ }^{\mathrm{a}, *}$, R.C. Arkin ${ }^{\mathrm{b}}, \mathrm{S}$. Jiang ${ }^{\mathrm{b}}$, M. O’Brien ${ }^{\mathrm{b}}$, F. Tang ${ }^{\mathrm{a}}$, P. Tang ${ }^{\mathrm{a}}$ \\ a Fordham University, New York, USA \\ ${ }^{\mathrm{b}}$ College of Computing, Georgia Institute of Technology, Atlanta, GA, USA
}

\section{H I G H L I G H T S}

- This paper addresses the challenges involved in building a software tool for automatically verifying the behavior of multi-robot waypoint missions using formal methods.

- Missions can include uncertainly located obstacles and uncertain environment geometry as well as uncertainty in robot motion.

- We leverage a unique approach, VIPARS, to verifying performance guarantees for autonomous behavior-based robot software based on a combination of static analysis and Bayesian networks.

- Two approaches to modeling probabilistic localization for verification are presented: a high-level approach and an approach that allows run-time localization code to be embedded within verification.

- Verification and experimental validation results are presented for several autonomous robot missions, demonstrating the accuracy of verification and the mission-specific benefit of localization.

\section{A R T I C L E I N F O}

\section{Article history:}

Received 10 November 2016

Received in revised form 7 May 2017

Accepted 2 July 2017

Available online 17 July 2017

\section{Keywords:}

Probabilistic verification

Validation

Multi-robot missions

Behavior-based robots

\begin{abstract}
A B S T R A C T
Establishing a-priori mission performance guarantees is crucial if autonomous robots are to be used with confidence in missions where failure could incur high costs in life and property damage. Automatic mission software verification, in addition to simulation and experimental benchmarking, is a key component of the solution for establishing performance guarantees. This component requires automatically verifying that the software constructed by the mission designer when executed in a partially known environment will adhere to the performance guarantee. In prior work we developed VIPARS, a unique approach to verifying performance guarantees for autonomous behavior-based robot software based on a combination of static analysis and Bayesian networks. While that approach produced fast and accurate verification of single robot missions with robot motion uncertainty, it did not address multiple-robot missions or any form of uncertainty related to environment geometry.

This paper addresses the challenges involved in building a software tool for verifying the behavior of a multi-robot waypoint mission that includes uncertainly located obstacles and uncertain environment geometry as well as uncertainty in robot motion. An approach is presented to the problem of a-priori specification of uncertain environments for robot program verification. Two approaches to modeling probabilistic localization for verification are presented: a high-level approach and an approach that allows run-time localization code to be embedded in verification. Verification and experimental validation results are presented for several autonomous robot missions, demonstrating the accuracy of verification and the mission-specific benefit of localization
\end{abstract}

(C) 2017 Elsevier B.V. All rights reserved.

\section{Introduction}

It is crucial to be able to establish an a-priori guarantee of mission success for robots deployed in critical missions such as

\footnotetext{
This research is supported by the Defense Threat Reduction Agency, Basic Research Award \#HDTRA1-11-1-0038.

* Corresponding author.

E-mail address: dlyons@fordham.edu (D.M. Lyons).
}

counter weapons of mass destruction (C-WMD) and other missions where failure brings serious consequences to life and property. In other, less critical applications it is highly desirable to have apriori guarantees of performance to reduce overall mission costs. In prior work for the Defense Threat Reduction Agency (DTRA) [1], we have developed an approach to automatic verification of performance guarantees for autonomous behavior-based robot mission software operating in uncertain environments. We developed a 
unique combination of static analysis and Bayesian networks for efficient and automatic verification of performance guarantees for missions developed in the MissionLab [2] robot mission design toolkit, and demonstrated by experimental validation that the approach produced trustworthy results. While that work detailed the foundation of the approach, it only addressed the single-robot scenario, and it assumed operation in an open space, with no unexpected obstacles. This paper leverages that prior work [1] to also address the challenges of automatic verification of performance guarantees for single and multi-robot missions in environments with uncertain geometry.

Verification of robot software is related to general purpose software verification in its objective of taking a program as input and automatically determining whether that program achieves a desired objective or not [3]. It differs in that a robot program continually interacts with its uncertain and dynamic environmentwhich therefore must be included as part of the verification problem. In fact, this is rarely done in robot program verification and was one of the novel contributions of our prior work [1]; so, rather than addressing computational verification problems such as absence of deadlock or absence of run-time errors [4,5] (important, but typically addressed in software verification), we have focused on establishing performance guarantees for the mission software with a complex and uncertain environment model. Also, like [6], we have focused on verification of behavior-based autonomous robots, a modular approach capable of robust performance in uncertain environments.

One contribution of this paper is an approach to the problem of a-priori specification of uncertain environments for robot program verification, in particular, to specifying an environment which may or may not contain obstacles with locations specified probabilistically. A consequence of this environment model is that verification must consider variable values that result from the robot encountering an obstacle at some location with some probability and not encountering the obstacle there. Therefore, a second contribution is a novel method to extend the Bayesian Network formulation of [1] to reason about random variables with different subpopulations.

We also apply our technique to a behavior-based robot program that includes probabilistic localization using the Adaptive Monte Carlo Localization algorithm (AMCL) running under ROS [7]. This the first time, to our knowledge, that a formal V\&V method has been applied in this way. Verification of this application is challenging because it absolutely requires an environment model, separate from, and interacting with, the behavior-based software. The model has to include the physical location of the robot, the geometry of the map, and the relationship between these and the sensor measurements. A third contribution of the paper are models for including localization in the verification process: a high-level, idealized model and a model with specific localization (or any probabilistic) software.

An important aspect of our work has been backing up our verification results by extensive, experimental validation. Rather than just presenting the results of verifying mission software for all the missions in this paper, we compare these verification results with performance statistics from experimental validation trials.

The next section reviews the literature in verification of robot software. Section 3 is a sufficient review of the foundational material from [1] as a basis for the new contributions. Section 4 addresses a multi-robot mission that may encounter obstacles, while Section 5 presents and compares two approaches to verifying a mission with probabilistic localization software. In each case, experimental validation is used to demonstrate that the verification results are consistent with real performance statistics. Section 6 summarizes and discusses our novel contributions and future work.

\section{Literature}

Formal verification can be used as a design tool to determine whether a piece of robot software will function as desired without having to execute the software physically. The field has made significant strides in recent years with the development of modelchecking [3] and SMT engines [8]. However, formal verification can at best produce an approximation of robot performance, due to the undecidability of the underlying verification problem. A crucial issue in selecting a verification approach is to understand what aspects of the robot software problem to focus on and how to leverage these to yield efficient automatic verification tools.

Behavior-based robot programming is an important design approach in autonomous robotics because it yields programs that are robust to uncertainty about exactly what environment the robots will face during execution. For this reason, verification of behaviorbased robot programs is being addressed by some researchers, e.g., $[6,9,10]$, and we also focus on that approach here.

Many robot software verification papers do not include any model of the environment in which the mission is carried out, verifying properties of the software itself such as absence of deadlock or run-time errors $[4,5]$. Such an approach might verify that a robot never issues a collision velocity, but not that a robot might roll or be mistakenly pushed into an obstacle-actions that only take place within the environment model. Or it might verify that a bombdisposal robot has snipped the power wire (the robot's action), but not that the bomb itself has not exploded (a function of the separate state of the bomb).

In some cases, the properties to be verified are used themselves to implicitly express the designer's knowledge (or expectation) of environment dynamics [11]. A simple example of this is assuming that testing for a motor stall is the same as testing for a collision. This informal approach is an error-prone way to capture environment dynamics; a stall might be caused by factors other than a collision.

Some of the most recent verification work does include environment models: The UK EPSRC-funded project on Trustworthy Robotic Assistants proposed representing unstructured environments using the Brahms [12] agent modeling language; however, while this does model environment dynamics, it does not address the crucial issues of motion and sensing uncertainty. These uncertainties can be the difference between success and failure for a critical mission. The latter was identified in [5] as one of the key 'lessons learned' in applying standard formal techniques to robot missions. Fisher et al. [13] address the difficulty of specifying a-priori conditions by verifying the robot's belief rather than its actual behavior. However, the robot's belief may not correspond to what actually happens. In an alternate approach, Guo et al. [14] and Sarid et al. [15] both iteratively produce a correct by construction program as uncertain information becomes known. However, it is not possible with that approach to verify the program in advance.

A common approach to verification is to manually implement the algorithm to be verified in a formal framework. For example, in Proetzsch et al. [16] the robot software to be verified is written in the verification language Quartz. Kim et al. write their robot software to be verified in Esteral [11]. Of course, this reimplementation may not represent the actual software; Published descriptions, even for widely known algorithms, have been shown to contain errors [17]. It also means that verification requires a huge investment of expertise and manpower to rewrite existing robot software into the verification framework [11]. We take a different approach: Mission designers work directly in the MissionLab design toolkit, and their software can be automatically translated to PARS [18] for verification-they never have to deal with the formal framework themselves and just use their regular tools for robot mission construction. 
Kiekbusch et al. [6] address automatic verification of behaviorbased software in their $i B 2 C$ framework. As with our MissionLab approach, their software is automatically translated to a verification framework-a set of finite state automata for model-checking. They also provide some environment modeling in the form of scenarios which are specific configurations of the environment for testing purposes. However, due to the state explosion problems of their model-checking tool, they can only verify binary behavior activation conditions such as whether an obstacle avoidance behavior is active, rather than the actual motions of the robot in response to the obstacle. They do not represent uncertain information and simply list the scenarios they wish to test against.

Generally-related work to ours also includes correct-byconstruction methods for teams of robots, and verification and validation of planning and scheduling systems. The former focus on automatic synthesis [19], not verification, of a program. In the latter, where a domain model is used to make a plan or schedule to achieve a high-level goal, "experience has shown that most errors are in domain models" [20]-which can only be checked if a separate environment model is included in verification. The work reported in this paper addresses verification using an explicit uncertain environment model.

\section{Designing robot missions with verification}

This section briefly reviews the material from [1] as a basis for a standalone, self-contained presentation of the new contributions in this paper. The first subsection is an overview of the programming toolkit for designing robot mission software, MissionLab, and the way in which automatic verification is added to this toolkit. The next subsection introduces the formal framework PARS (Process Algebra for Robot Schemas) used in verification. The final subsection reviews the verification framework itself, a combination of static analysis and Bayesian networks.

\subsection{Mission design}

MissionLab is a usability-tested [21] graphical programming toolkit for robot missions, including graphical editor, mission simulation and execution logging capabilities among others. The mission designer constructs the mission using MissionLab, as illustrated in Fig. 1.
The VIPARS (Verification in PARS) [1] module is designed to work with MissionLab and provide a performance verification functionality. VIPARS module inputs include:

- the mission program, as designed in MissionLab's CfgEdit graphical interface;

- a set of designer selected library models of the robot, and sensor systems;

- the mission operating environment; and,

- the mission performance criteria.

A mission designer could, for example, construct a single-robot, waypoint mission, indicate that it will take place in a moderatelycluttered warehouse environment, and that it will be performed by a Pioneer 3-AT robot equipped with sonar and gyroscope. She could then choose performance criteria that fit the mission (for example, that the robot moves within at least $0.1 \mathrm{~m}$ of each waypoint and finishes all waypoints in under $100 \mathrm{~s}$ ). She can then use VIPARS to verify whether or not the mission will always meet this performance criterion with some given threshold probability.

Prior to the VIPARS module, the mission software is automatically translated to PARS [18] a formal, process-algebra language. The library models of Pioneer 3-AT, sonar and gyroscope, and moderately cluttered indoor environment are then combined with the mission software to generate a single PARS system which will be analyzed for the performance guarantee. Our intent is that these robot, sensor and environment models are used, but not constructed, by the mission designer; they are built in PARS as probabilistic process models parameterized with robot and sensor calibration data and provided to a designer with the verification module.

VIPARS verifies whether the mission software will achieve the specified performance criteria (typically spatial and temporal constraints) using the selected robot/sensors in the selected operating environment. It also generates predicted performance information that can be used by the designer to either improve the system performance or abort the mission to avert catastrophic failures. The verification component supports an iterative cycle for designing high-performance robot behavior for critical missions.

\subsection{PARS}

PARS is a process-algebra designed for the purpose of representing robot software, and the robot, sensor and environment

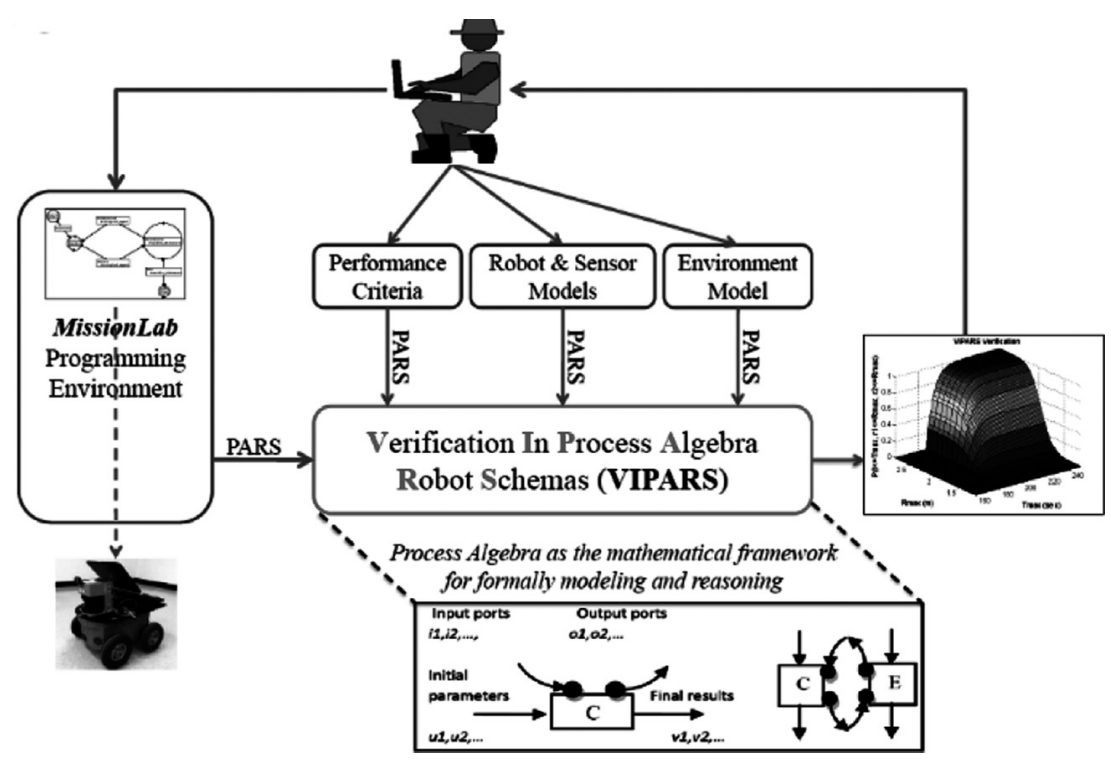

Fig. 1. MissionLab/VIPARS system architecture. 


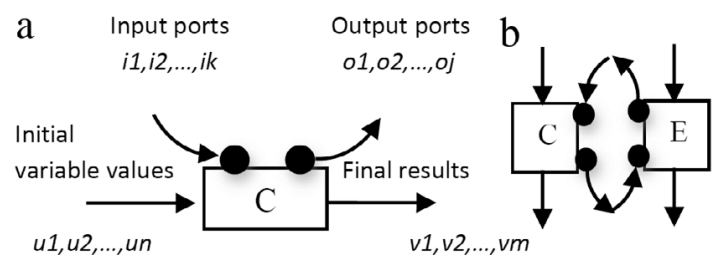

Fig. 2. (a) PARS process; (b) process network (from [1]).

models with which the software interacts. The algebraic syntax facilitates developing static analysis algorithms (algorithms that analyze programs without executing them) to identify the interactions between the robot program and its environment. Although PARS was designed for representing robot programs, and in particular robot schemas style, behavior-based programs [22], in fact it shares many characteristics with other process algebras such as CSP [23] and LOTOS [24] and has an operational semantics defined using port automata [25]. As such it could be used to represent any programs, and any robot programming style. However, our more specific results are focused on behavior-based programs because they have a structure that can be leveraged to address efficient verification.

Fig. 2 shows the PARS model of a process and process network. A process $\boldsymbol{C}$ (Fig. 2(a)) is written as:

$C\left\langle u_{1}, \ldots, u_{n}\right\rangle\left(i_{1}, \ldots, i_{j}\right)\left(o_{1}, \ldots, o_{k}\right)\left\langle v_{1}, \ldots, v_{m}\right\rangle$

where $u_{1}, \ldots, u_{n}$ are the (finitely many) initial variable values for the variables of the process, $i_{1}, \ldots, i_{j}$ and $o_{1}, \ldots, o_{k}$ are input and output port connections, respectively, and $v_{1}, \ldots, v_{m}$ are final result values generated by the process.

Processes are either atomic or composite. A process is defined as a composition of other processes as follows:

$$
\begin{aligned}
& \langle\text { processdef }\rangle::=\langle\text { process }\rangle{ }^{\prime}={ }^{\prime}\langle\text { processexpr }\rangle \\
& \langle\text { processexpr }\rangle::=\langle\text { processeq }\rangle \text { '|' }\langle\text { processeq }\rangle \mid \\
& \langle\text { processeq }\rangle \text { '\#’〈processeq }\rangle \\
& \langle\text { processeq }\rangle::=\langle\text { processexpr }\rangle \text { ';’'processexpr }\rangle \mid
\end{aligned}
$$

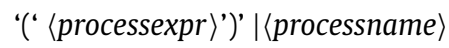

where '|' denotes parallel composition (parallel max), '\#’ disabling composition (parallel min), and ';' denotes sequential composition, and where $\langle$ process $\rangle$ and $\langle$ processname $\rangle$ are a bolded capital letter or word.

For example, the parallel composition:

$$
\mathbf{S}=\mathbf{C}(c 1)(c 2) \mid \mathbf{E}(c 2)(c 1)
$$

specifies two parallel processes $\mathbf{C}$ and $\mathbf{E}$ as shown in Fig. 2(b), with the input and output ports connected correspondingly. The labels $c 1$ and $c 2$ are called port connection labels and their purpose is to specify the connection map between the ports of the parallel processes.

Each process that terminates can terminate in either a stop or an abort condition. There is no separate 'choice' operator in PARS. However, a process that evaluates a condition $c$ is defined to terminate in a stop status if $c$ and in abort if not $c$. A sequential chain of processes, such as $\mathbf{E q}\langle x, y\rangle$; $\mathbf{P}$, terminates for the first process in the chain that has a termination condition of abort (e.g., if $x \neq y, \mathbf{P}$ is not reached because $\mathbf{E q}$ aborts).

Repetitive computation (e.g., loops) is modeled by a tailrecursive (TR) process definition, written for example:

$\mathbf{P}\langle x\rangle=\mathbf{Q}\langle x\rangle\langle y\rangle ; \mathbf{P}\langle y\rangle$.
Eq. (2) defines a process $\mathbf{P}$ that repeats process $\mathbf{Q}$ until $\mathbf{Q}$ aborts, at which point $\mathbf{P}$ terminates, returning its results. In this example, the process $\mathbf{Q}$ is the body of the TR process, similar to the body of a loop.

A flow function $f_{P}\left(u_{1}, u_{2}, \ldots, u_{n}\right)=\left(v_{1}, v_{2}, \ldots, v_{m}\right)$ is associated with each $\mathbf{P}$, mapping the values of the variables of $\mathbf{P}$ at the start to those at the end. The flow-function for atomic processes are specified a-priori, and those for a composite process can be built up from the flow functions of its components, e.g., for $\mathbf{T}\langle x\rangle\langle z\rangle=$ $\mathbf{P}\langle x\rangle\langle y\rangle ; \mathbf{R}\langle y\rangle\langle z\rangle$ we can say $f_{T}(x)=f_{R} \circ f_{P}(x)$ if $\mathbf{P}$ does not abort. Static analysis algorithms to calculate flow functions play a key role in VIPARS verification.

\subsection{Verification in PARS (VIPARS)}

The robot mission software is converted to PARS [18] and combined with the PARS definitions for the robot, sensor and physical environment models (selected by the user) producing a parallel network Sys of communicating processes. For example, a robot controller Ctr with variable $r_{1}$, and an environment model ${ }^{1}$ Env with variable $r_{2}$, would be written as:

$\operatorname{Sys}\left\langle r_{1}, r_{2}\right\rangle=\mathbf{C t r}\left\langle r_{1}\right\rangle(a)(b) \mid \operatorname{Env}\left\langle r_{2}\right\rangle(b)(a)$.

In the example of Eq. (3), the input of $\mathbf{C t r}$ (sensor signals) is connected to the output of Env, (a), and the input of Env is connected to the output (control signals) of Ctr, $(b)$, similar to the process network in Fig. 2(b). If Eq. (3) were a sequential composition like Eq. (2) then we could extract flow functions for the combined interaction of controller and environment and use this function as the basis for verifying all possible executions of the system. However, the addition of port communication complicates the relatively simple definition of flow functions! The flow function associated with a process no longer just depends on the variables of that process, but could depend in a complex way on variables and computations of other parallel processes. To address this, a constraint on the form of parallel compositions is leveraged, namely that all processes are written as tail-recursive (TR) processes. This does not restrict what can be computed but allows us to propose a special static analysis approach to efficiently verifying all possible executions of a behavior-based systems.

In behavior-based robot software, such as that produced by MissionLab, sensory information is continually being inspected to determine which behaviors should be activated and how to parameterize them. The software is looking for affordances in the environment that will further the objectives for the mission-as a simple example: moving toward goal locations, but away from obstacles. The intuition is that a behavior-based system has behavioral 'states' each with an associated set of sensory triggered responses.

This is modeled here as a parallel composition of TR processes representing ongoing behaviors or the monitoring of affordances. When a behavior terminates or when an affordance is detected, additional behaviors or affordance monitoring may be added to the parallel composition.

Leveraging the TR structure, an interleaving theorem ${ }^{2}$ is presented in [1] to convert processes of the form of Eq. (3) to a sequential form as shown in Eq. (4) below. The intuition here is that the set of TR process bodies can be composed into a single system TR body called the system period, shown as the process Sys'

\footnotetext{
1 As a verbal shortcut, we will include the models of the robot, and sensors in the term environment model.

2 In process algebra, an interleaving theorem relates the sequential and parallel composition operations.
} 
in Eq. (4), and similar to the concept of a hyper-period ${ }^{3}$ in process scheduling.

$$
\begin{aligned}
\operatorname{Sys}\left\langle r_{1}, r_{2}\right\rangle & =\mathbf{C t r}\left\langle r_{1}\right\rangle(a)(b) \mid \operatorname{Env}\left\langle r_{2}\right\rangle(b)(a) \\
& =\operatorname{Sys}^{\prime}\left\langle r_{1}, r_{2}\right\rangle\left\langle r_{1}^{\prime}, r_{2}^{\prime}\right\rangle ; \operatorname{Sys}\left\langle r_{1}^{\prime}, r_{2}^{\prime}\right\rangle \\
f_{\text {Sys' }}\left(r_{1}, r_{2}\right) & =\left(f_{\text {Sys', }, 1}\left(r_{1}, r_{2}\right), f_{\text {Sys', }, 2}\left(r_{1}, r_{2}\right)\right) \\
& =\left(r_{1}^{\prime}, r_{2}^{\prime}\right) .
\end{aligned}
$$

A static analysis algorithm Sysgen was developed based on this interleaving theorem to construct the system period, Sys' in Eq. (4), given the processes, connections and communications in Sys. This construction reduces the state explosion of all orders of a set of parallel processes to the single interleaving of the system period.

Once Sysgen analysis is complete, a system flow function can be extracted from Sys'. In the small example of Eqs. (3), (4) above, the function extracted is shown in Eq. (5). This is a recurrent function that evaluates the new values for $r 1$ and $r 2$ as computed by the interactions between $\mathbf{C t r}$ and $\mathbf{E n v}$ in each execution of the system period Sys'.

Process variables, such as $r_{1}, r_{2}$ in the example above, can be random or deterministic variables. Typically, mission software variables are deterministic. However, variables in robot, sensor and environment models can be random representing uncertainty associated with their values. To include both random and deterministic cases, flow functions, which relate variable values at recursion step $i$ of Sys' to those at $i+1$, can be written as conditional probabilities, e.g.:

$f_{\text {Sys' }, r 1}\left(r_{1, t}, r_{2, t}\right)=P\left(r_{1, t+1} \mid r_{1, t}, r_{2, t}\right)$.

In the final phase of VIPARS processing, extracted flow functions are converted to conditional probabilities. Random variables are represented as multivariate Mixtures of Gaussians, and operations on random variables are automatically translated by VIPARS into operations on distributions [26]. These are then the basis of a Dynamic Bayesian Network (DBN) [27] used to carry out forward propagation of probability distributions, to determine whether the combination of controller and environment will meet a performance specification.

Although [1] discusses more complicated performance guarantees, we will typically restrict our attention to the guarantee that a mission will achieve some criterion on environment variables (usually a spatial accuracy for a waypoint goal and/or a temporal requirement for achieving the mission) with probability greater than a threshold before a time-limit. We demonstrated that this approach is fast and accurate when validated against physical executions (most recently [28]).

\section{Multirobot mission with uncertain obstacles}

Bounding overwatch is a military movement tactic used by units of infantry to advance forward when crossing dangerous areas [29]. In the first mission we will address, a team of two robots will use this strategy to move stealthily inside a building to search for biohazards which may be guarded by hostile forces and in which they may encounter obstacles along their route.

Fig. 3 shows the bounding overwatch mission where two robots coordinate their movements in a "leapfrogging" manner while advancing toward a biohazard. Robot2 begins by bounding toward 01, the first Overwatch position. When it reaches 01, a "Cleared" message is sent to Robot 1 indicating that it is safe to proceed. Robot 1 then bounds to $\mathbf{0 2}$ and sends the "Cleared" message to Robot2, and so on. The mission ends with Robot 2 at $\mathbf{0 7}$, near the biohazard. The operating environment of this mission includes

$\overline{3}$ The LCM of all the task periods in a scheduling problem.

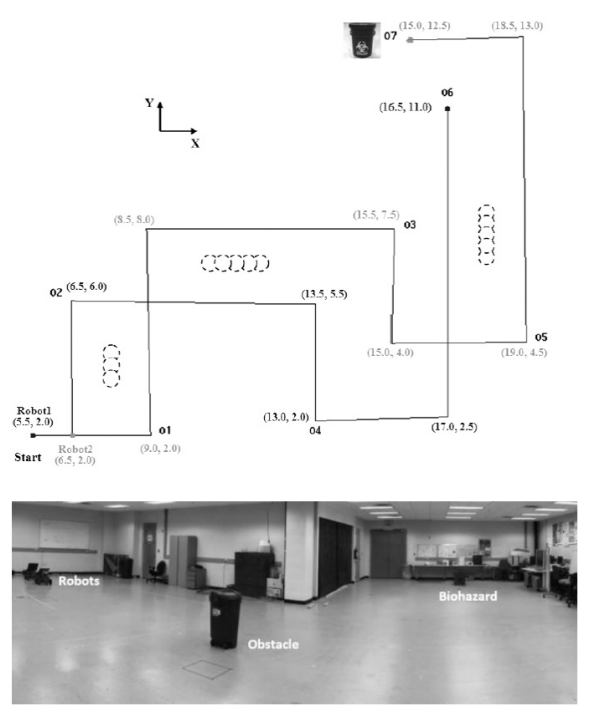

Fig. 3. Bounding overwatch with two robots: Map (top), operating environment (bottom).

some obstacles whose existence or exact locations are not known with certainty in advance; if they are present, the obstacles will be within the locations illustrated with dashed circles shown in Fig. 3. This lack of a-priori certainty about the environment geometry is a challenge for verification in efficiently representing and checking all the potential obstacle-related motions of both the robots.

\subsection{Bounding overwatch mission}

The behaviors of Robot 1 and Robot 2 are specified graphically in MissionLab as behavioral finite state automata (FSAs). Each behavioral FSA consists of the following behaviors:

- GoToGuarded: move to a waypoint while avoiding obstacles;

- NotifiedRobots: send a "Cleared" message to the other team members;

- Spin: rotate the robot;

- Stop: mission concluded;

- AtGoal: sensory trigger for arrival at location;

- HasTurned: sensory trigger for arrival at orientation;

- Notified: sensory trigger for "Cleared" message;

- MessageSent: sensory trigger for message sent.

The behavioral FSA of triggers and behaviors for Robot 1 is shown in Fig. 4 and that for Robot2 is similar.

The behavioral FSA is translated to a MissionLab internal language called CNL [2] and a translator from CNL to PARS [18] produces a process model of the program which includes a detailed implementation of all the behaviors and triggers. The following performance criteria are used to evaluate this mission performance:

Success $=\left(r_{1} \leq R_{\max }\right)$ and $\left(r_{2} \leq R_{\max }\right)$ and $\left(t \leq T_{\max }\right)$

where $r_{1}$ and $r_{2}$ are Robot1's and Robot2's relative distances to their respective goal and $t$ is the mission completion time, where $R_{\max }$ is the success radius, and $T_{\max }$ is the maximum allowable mission time. The bounding overwatch mission is only considered successful when both robots are within $R_{\max }$ radius of their respective goal locations and when they complete the mission in under $T_{\max }$ seconds. 


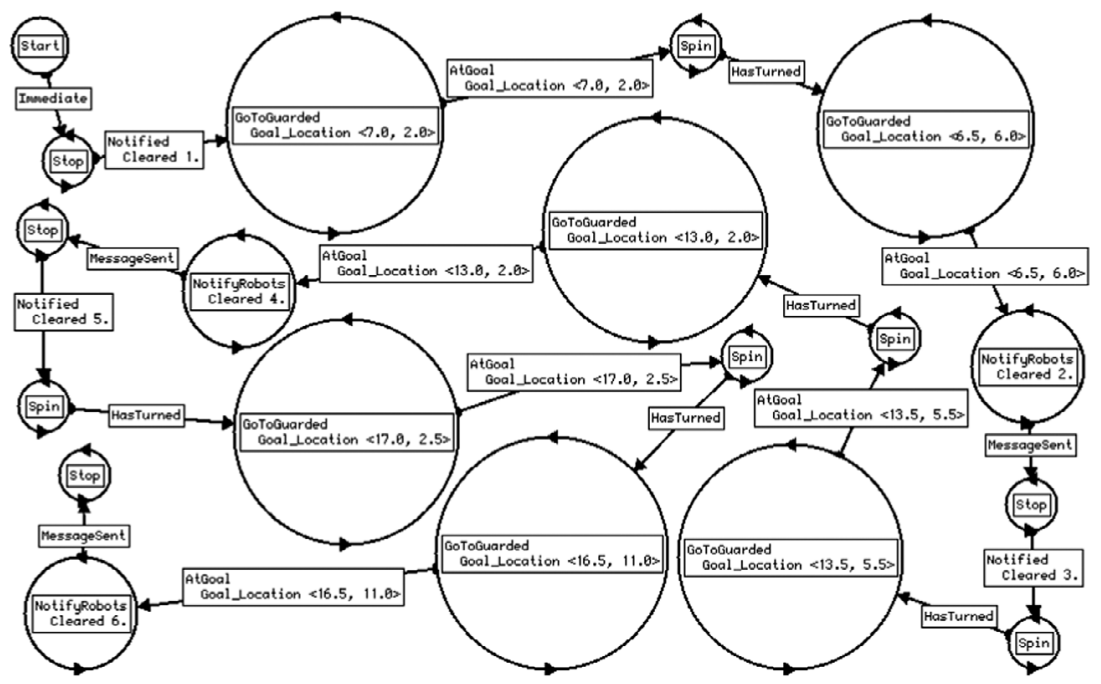

Fig. 4. Behavioral FSA for Robot1.

\subsection{Robot motion model}

In [1] we presented a PARS robot process model Robot with motion and position sensing uncertainty.

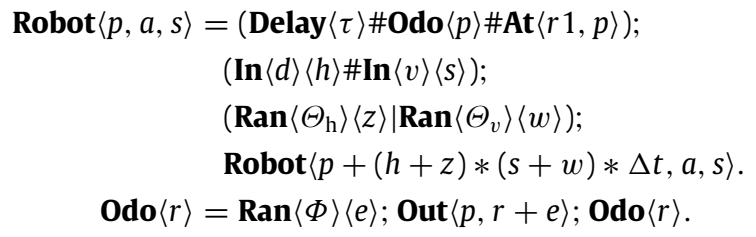

Robot accepts a unit vector heading input on port $d$ or a speed in the direction of the heading on port $v$. The process $\mathbf{A t}\langle r 1, p\rangle$ represents robot $r 1$ at location $p$. The process Odo (Odometry sensor) makes position information (with noise $e \sim \Phi$ ) available in a loop on port $p$ until terminated by the timer process Delay. The new position of the robot is calculated as the old position $p$ incremented by a speed $s$ with added noise $w$ in the direction of the commanded heading $h$ with added noise $z$. The odometer position is the actual position with added noise $e$. The actuator and odometer noise (the variables $z, w$, and $e$ ) are characterized by the distributions for speed, heading and sensor noise, e.g., $\Theta_{h}=$ $N\left(\mu_{h}, \sigma_{h}\right), \Theta_{v}=N\left(\mu_{v}, \sigma_{v}\right)$, and $\Phi=N\left(\mu_{m}, \sigma_{m}\right)$. The flow function for the position variable of the robot model, with operations on random variables translated to operations on distributions, is

$p_{t+\Delta t}=p_{t} \cdot\left(\Theta_{h} \cdot h_{t+\Delta t}\right) \cdot\left(s_{t} \cdot \Theta_{v}\right) * \Delta t$,

where ' $'$ ' denotes convolution.

The robot model used in this paper follows this same structure but is more detailed in its representation of the motion uncertainty. The new robot position distribution $p_{t+\Delta t}$ is calculated as the old position distribution $p_{t}$ convolved with $s_{t+\Delta t} h_{t+\Delta t} \Delta t-$ a nominal change at speed $s_{t}$ with heading unit vector $h_{t}$ for time $\Delta t$ and a motion uncertainty term. The latter is a convolution of a translational TX, rotational TR and skitter TS uncertainty component:

$$
\begin{aligned}
\operatorname{TX}\left(s_{t+\Delta t}, h_{t+\Delta t}\right)= & X \cdot \frac{s_{t+\Delta t}}{s^{k}} \cdot H\left(s_{t+\Delta t}-1\right) \cdot R\left(h_{t+\Delta t}\right) \cdot \Delta t \\
\operatorname{TR}\left(s_{t+\Delta t}, h_{t}, h_{t+\Delta t}\right)= & U \cdot\left|h_{t}-h_{t+\Delta t}\right| \\
& \cdot\left(2 H\left(\theta_{t}-\theta_{t+\Delta t}\right)-1\right) \cdot R\left(h_{t+\Delta t}\right) \cdot \Delta t \\
T S\left(s_{t+\Delta t}, h_{t}, h_{t+\Delta t}\right)= & W \cdot\left|h_{t}-h_{t+\Delta t}\right| \cdot H\left(1-s_{t+\Delta t}\right) \\
& \cdot\left(2 H\left(\theta_{t}-\theta_{t+\Delta t}\right)-1\right) \cdot R\left(h_{t+\Delta t}\right) \cdot \Delta t
\end{aligned}
$$

where
- $X, U$ and $W$ are robot specific bivariate normal distributions calibrated by multiple measurements over a range of distances and angles for each robot at calibration speed $s^{k}$.

- $R(h)$ is an operator that rotates a bivariate distribution by the unit vector $h$.

- $H(x)$ is the Heaviside step function.

- $\theta_{t}$ is the angle of heading unit vector $h_{t}$.

In summary, the flow function for the position distribution $p_{t+\Delta t}$ is now:

$p_{t+\Delta t}=p_{t} \cdot\left(s_{t} h_{t+\Delta t} \Delta t\right) \cdot(T X \cdot T R \cdot T S)$

\subsection{Multiple robots}

This mission uses two robots, and the two robots interact directly to synchronize their motion (Fig. 4) and indirectly as physical obstacles to each other. Multiple robots are added to the environment model by creating extra instances of the Robot process, each with unique $X, U$ and $W$ calibration distributions measured by calibration experiments on a physical robot. Each robot process has its own input and output ports through which the mission software can communicate. No extension to the process algebra model was required to handle the multiple robot case.

The direct communication between the robots is modeled for verification by a simple, centralized communication structure. The Notify and MessageSent behaviors map to port read and write commands from the PARS translation of Fig. 4 (and its equivalent for Robot2) to a single communication process Comm. No message transmission latency or error was modeled for this example.

\subsection{Uncertain geometry model}

Since the geometry of the environment is not completely known in advance, we construct a probabilistic model that includes whatever a-priori information there is. One way to generate such a model is as shown in Fig. 5: Several spatial locations along the mission are annotated a-priori as being potential obstacles; this is the approach we will take in this mission. Another approach would be to use the map output from probabilistic mapping software that has been used to measure the environment-we will take that approach in a later section. Of course, both approaches could be combined.

For this mission, the physical environment is modeled as a collection of isotropic bivariate Gaussian mixtures: Fig. 5(a) shows a 
a

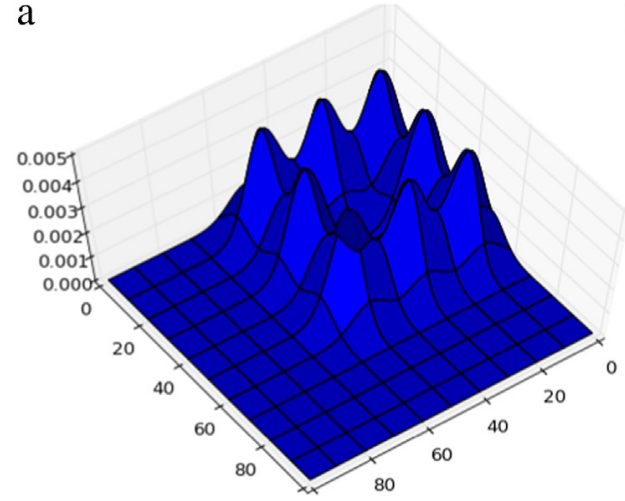

b

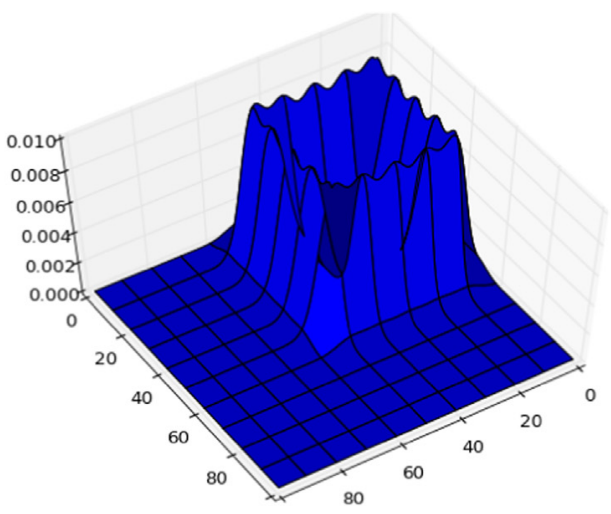

Fig. 5. Modeling geometry with bivariate Gaussian mixtures.

mixture of 8 members modeling a rectangular 2D obstacle. Fig. 5(b) shows the model with 16 members.

The GoToGuarded behavior in MisssionLab is translated to the process network shown in (9) which implements its behavior:

$\operatorname{Coop}\langle 1,1,1\rangle(v g, v o, v n)(v) \mid$

Move_to $\langle P O, G 3\rangle(p R)(v g) \mid$

Noise $\langle n s\rangle(p R)(v n) \mid$

Avoid_Obstacles $\langle r\rangle(p R, o b R)(v o)$.

The Avoid_Obstacles process inputs robot position (through connection $p R$ ) and any sensed obstacles (on $o b R$ ) and generates a potential-field based avoidance velocity output (vo) [22]. Move_to generates a velocity toward a waypoint $G 3(v g)$ and Noise generates a small velocity perturbation to escape potential minima $(v n)$. The Coop process combines all three vectors into a single command velocity $(v)$ with equal weights $(1,1,1)$.

In execution, the input and output of these processes correspond to the connections of GoToGuarded with the real robot and its sensors, and through these, with the actual execution environment. However, in verification, this information is provided instead by robot, sensor and physical environment models selected by the user. For this mission, these are shown below:

$\operatorname{Robot}\langle P 0, \Delta \boldsymbol{t}, \boldsymbol{\varphi}\rangle(v)(p R) \mid$

Sensors $\langle S 0, s r, s n\rangle(p R, p E)(s) \mid$

Geometry $\langle E\rangle(p R, p R 2)(p E)$.

The Robot process takes a velocity command (on $v$ ) and generates a new position distribution (on $p R$ ) according to (8) and where $p_{t}, v_{t}$ $\sim \operatorname{MG}\left(M_{p}\right)$ are modeled as mixtures of bivariate Gaussians representing the 2-D location and velocity of the robot. The Sensors process calculates what obstacle locations will be sensed by the robot, implemented as follows:

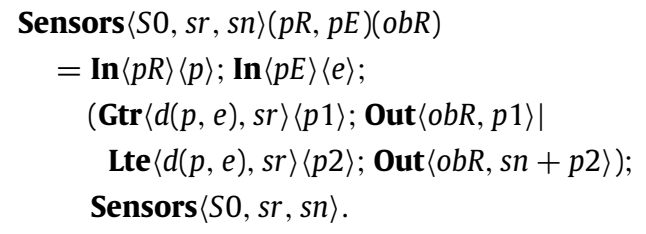

The robot position (on $p$ ) and geometry (on $e$ ) are inputs from whatever Sensors has been connected to-in this case, the Robot process and the Geometry process. Geometry continually adds the latest position distributions for both robots to the static geometry distribution (obstacles) and transmits this to the Sensors process (e). This approach handle the indirect interaction between robots, and it generalizes linearly to any number of robots.

The distance function $d(p, E)$ calculates what portion of the environment is within the sensor range $(s r)$. The procedure for
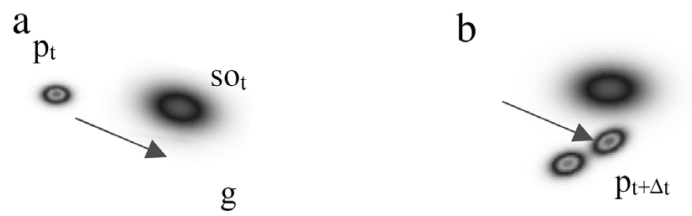

Fig. 6. Example of obstacle avoidance.

determining potential collisions and sensor feedback involves computing the Bhattacharyya Coefficient [30] between robot position and the geometry distribution. This coefficient measures the amount of overlap between two multivariate normal distributions as follows:

$$
\begin{aligned}
& B C\left(N\left(\mu_{0}, \Sigma_{0}\right), N\left(\mu_{1}, \Sigma_{1}\right)\right) \\
& \quad=\exp \left(-\frac{1}{8}\left(\mu_{0}-\mu_{1}\right)^{T} \Sigma^{-1}\left(\mu_{0}-\mu_{1}\right)\right) \sqrt{\frac{\sqrt{\left|\Sigma_{0}\right|\left|\Sigma_{1}\right|}}{|\Sigma|}} \\
& \text { where } \Sigma=\frac{\left|\Sigma_{0}\right|\left|\Sigma_{1}\right|}{2}
\end{aligned}
$$

$d(p, e)$ generates a bivariate distribution with members corresponding to the joint probabilities between the members of the $\mathrm{p}$ and e variables. The result of sensing $(o b R)$ is this distribution (convolved with a sensor noise distribution $(s n)$ ).

\subsection{Conflicting hypothesis histories}

The flow functions automatically extracted by VIPARS from the GoToGuarded network (9) connected to the environment model (10) include the effects of condition processes (such as Gtr and Lte) and can be written in terms of the Heaviside step function $H($.) and unit vector $u($.). Operations on variables (e.g., addition) are translated to equivalent operations on distributions (e.g., convolution). The following are among the flow functions extracted and just come from the definition of the GoToGuarded behavior:

$f_{v o}(s, p)=r-H\left(r-s o_{t}\right) s o_{t}$,

$f_{v g}(p, g)=u\left(p_{t}-g\right) s o_{\max }$

$f_{v}\left(v_{o}, v_{g}, v_{n}\right)=v_{0} \cdot v_{g} \cdot v_{n}$.

The obstacle velocity (vo in (9)) is specified by $f_{v o}$ as linearly proportional to the distance to the obstacle $r-s o_{t}$ but at most $r$ if there are obstacles seen. The goal velocity $f_{v f}$ is a fixed velocity $s_{\max }$ in the direction of the goal $u\left(p_{t}-g\right)$. (In fact, there is a ramp-down to the goal, omitted here for simplicity.) The final velocity is just the convolution of the noise, obstacle and goal velocities (the result of Coop in (9)). 
Consider the example shown in Fig. 6: At some time $t$, the position $\left(p_{t}\right.$, a single member distribution) is close enough to a sensed obstacle $s_{t}$ that an obstacle repulsive velocity $(v o)$ is generated in addition to the velocity toward the goal ( $v g$ ) (Fig. 6(a)). The portion of the position distribution that resulted in no obstacle detection ( $p 1$ in (11)) should be convolved with just a forward velocity; the portion that had obstacle detection ( $p 2)$ should be convolved with both forward and repulsion (Fig. 6(b)), capturing both potential outcomes.

In fact, however, there is insufficient information in the random variable model used by VIPARS to correctly represent this situation. During forward propagation of probability by the DBN, the information of sensor returns where collisions are predicted becomes separated from the information about which robot locations generated those returns. Informally: the $p_{t}$ mixture could be considered as a weighted collection of (Normal distribution) hypotheses for the robot position. The sensory data is generated from this list, but the correspondence between a sensory data mixture member, which originates from $s o_{t}$, and the hypotheses in $p_{t}$ that generated the member can be complicated:

(1) If the geometry $g$ is a multimodal distribution (almost certainly would be), then each member of $p_{t}$ will generate at least as many modes within $s o_{t}$ due to (11).

(2) The conditional nature of $f_{v o}$ (i.e., the step-function) means that not every member of $s_{t}$ generates a repulsive velocity (e.g., because it is too far away).

(3) The final, convolution for $f_{v}$ in (13) will apply goal and repulsion velocities to all position modes, not just the ones as shown in Fig. 6.

\subsection{Colored mixture of Gaussians (CMG)}

The solution to this dilemma is to allow subpopulations of the location variable to be tagged, and for this tag to be propagated to the sensing distribution, so that it becomes clear how the sensing relates to position. The mixture representation for random variable is extended as follows.

Definition. A colored mixture of Gaussians (CMG) is a mixture of Gaussians distribution in which each mixture member (mode) is tagged with a color label. If $a \sim C M G(C M)$, for $C M=$ $\left\{\left(\mu_{i}, \Sigma_{i}, w_{i}, c_{i}\right) \mid i \in 1 \ldots m\right\}$ the set of the mixture parameters (means, variances weights, and colors respectively), then $a_{i}$ will refer to $N\left(\mu_{i}, \Sigma_{i},\right), w\left(a_{i}\right)=w_{i}$ and $c\left(a_{i}\right)=c_{i}$. The mixture size is written $|a|=m$. A CMG is evaluated at a point $x$ in the usual way as $C M G(x ; C M)$ :

$$
\begin{array}{r}
C M G\left(x ;\left\{\left(\mu_{i}, \Sigma_{i}, w_{i}, c_{i}\right) \mid i \in 1, \ldots, m\right\}\right) \\
=\sum_{i=1}^{m} w_{i} N\left(x ; \mu_{i}, \Sigma_{i}\right), \quad \sum_{i=1}^{m} w_{i}=1 .
\end{array}
$$

The color tags allow related subpopulations of the CMG to be similarly transformed. Operations on random variable can now be converted to color-respecting operations. A color-respecting convolution operation in $f_{v}$ of $(13)$ can be defined:

Definition. The color respecting convolution $r=p \otimes q, r, p, q \sim$ $C M G$ is defined using the notation of the CMG definition as: $r_{i}=$ $p_{j} * q_{k} \Leftrightarrow c\left(p_{j}\right)=c\left(q_{k}\right)$ with weights $w\left(r_{i}\right)$ adjusted accordingly.

As an example, let $p_{t}$ have two members, $p^{1}$ and $p^{2}$, and if there are two members of the geometry distribution, $o^{1}$ and $o^{2}$, then $s o_{t}$ will have four members, two with $c\left(p^{1}\right)$ and two with $c\left(p^{2}\right)$ transformed by the (unimodal) sensor uncertainty distribution $(\mathrm{sn})$. The color respecting convolution operation in $f_{v}(13)$ will result in four velocity members: one for $v_{g}$ and one for the sum of $v_{g}$ for $p_{1}$ plus the sum of the two $v_{0}$ with color $\left(p^{1}\right)$, and two similarly for $p_{2}$. If the step function in $f_{\mathrm{vo}}$ trims members from $s o_{t}$, the members of $v_{o}$ and $v_{g}$ can still be correctly matched by color.

With this modification to the random variable framework of VIPARS - namely, the addition of color tags to the multivariate mixture model, and the extension of random variable operations (not just convolution) to respect color - the uncertain geometry model can be used to verify multirobot missions that include obstacle avoidance strategies. The next section presents evidence for this.

\subsection{Verification and validation}

The Overwatch mission presented in Section 4.1 is verified using the modified CMG filtering and the verification results experimentally validated in this section. In the interest of providing more than just a binary verification result, VIPARS produces a graph of the probability of mission success versus time (Time Criterion graph) and graph of the probability of final positional accuracy (Spatial Criterion Graph).

\subsubsection{Mission validation}

Each validation run consists of real robots carrying out the Overwatch mission. The operating environment of the mission is an indoor lab environment with tile floor. The biohazard is represented by a bucket marked with the biohazard symbol. The obstacles are trashcans with radii of approximately $0.25 \mathrm{~m}$. The dashed circles in Fig. 3 represent the potential locations of the obstacles. The number of obstacles (i.e., 1-3) and their locations are varied for each validation run, to reflect the uncertainty of their presence in the environment. At the end of each validation run, the following measurements relating to the performance criteria $R_{\max }$ and $T_{\max }$ are recorded:
1. $\boldsymbol{r}_{\mathbf{1}}-$ Robot1's relative distance to its goal location;
2. $\boldsymbol{r}_{2}$-Robot2's relative distance to its goal location;
3. $\boldsymbol{t}$-Mission completion time.

The complete validation experiment consists of 100 trials (calculated to cover all obstacle locations uniformly). The result of the validation experiment is compared to the verification result in the following subsection. These two results were generated without knowledge of each other and only compared after each was completed.

\subsubsection{Comparison of verification and validation results}

Besides generating accurate results, how to present verification results (i.e., performance guarantees) to the mission designer is also an important research question. We present a preliminary representation that consists of two steps: (1) define performance guarantee as the probability of success (i.e., the probability of meeting a performance criterion) and (2) divide the success probability into confidence regions.

Fig. 7 shows the verification and validation spatial criteria for this mission as the probability that both robots are within $R_{\max }$ radius of their respective goal locations $P\left(r_{1} \leq R_{\max }, r_{2} \leq R_{\max }\right)$ versus $R_{\max }$. The graph has three regions based on VIPARS verification: (1) High Confidence (Unsuccessful), (2) Uncertain, and (3) High Confidence (Successful). The High Confidence (Unsuccessful) region is where VIPARS predicts a zero probability of success, informing the operator that she should abort the mission or modify mission parameters (e.g., use different robots) if the verification result is in this region. The High Confidence (Successful) region is where VIPARS guarantees success with probability 1.0.

The mission operator has a special interest in this region since she expects the robots would get it right the first time for mission 


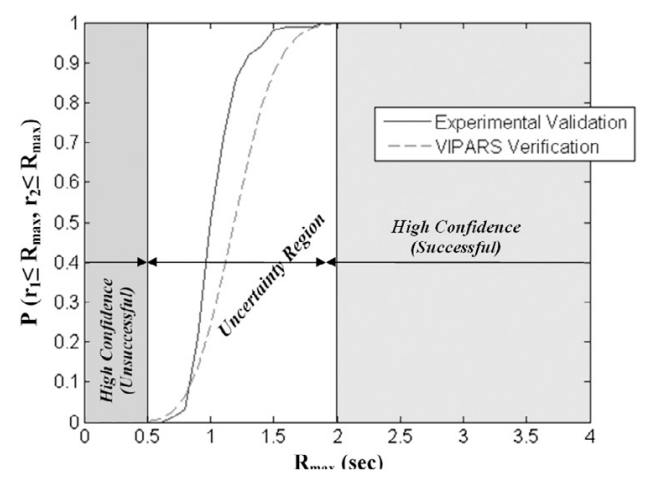

Fig. 7. Verification vs. validation of spatial criterion $P\left(r_{1} \leq R_{\max }, r_{2} \leq R_{\max }\right)$.

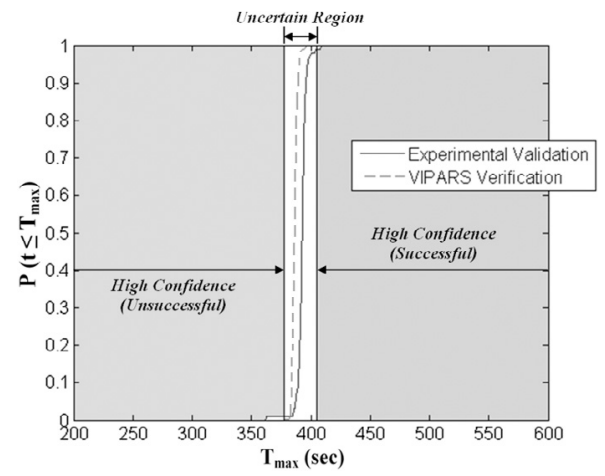

Fig. 8. Verification vs. validation of time criterion $P\left(t \leq T_{\max }\right)$.

requirements (e.g., $R_{\max }$ ) within this region. The region between High Confidence (Unsuccessful) and High Confidence (Successful) is defined as the Uncertain region, which corresponds to the region where the values of the VIPARS's mission success probability are between 0 and 1.0. In this region, the robots are not guaranteed to get it right the first time. Although both verification and validation curves are shown on Figs. 7 and 8, only the verification curve is used to define these regions.

Fig. 8 shows the verification and validation (performed over 100 trials as described) for the time criterion as a graph of the probability that the mission completes by $t, P\left(t \leq T_{\max }\right)$ versus $t$. The graph is again divided into the three confidence regions. We observed that most of the discrepancies between verification and validation are within the Uncertain region. The region is relatively small, and both validation and verification curves rise sharply, indicating that the boundary of $0 \%$ successful and 100\% successful is relatively sharp.

We also observed some discrepancies outside the Uncertain region, near its boundaries. Ideally, all the errors should be within the Uncertain region. However, the errors between the verification and validation success probabilities outside the Uncertain region are actually $\leq 0.01$ (i.e., within $\sim 1.01 \%$ error). At the boundary between Uncertain and High Confidence regions, VIPARS predicts a success probability of 1.0 while the actual experimental validation had a success probability of 0.9901 , which resulted in a verification error of 0.0099 . So, it is still justified to have a high confidence of mission success in the uncertain region since the experimental validation has a success probability of 0.99 and higher.

We have examined individual performance criterion separately thus far. However, the overall mission success was defined in terms of both spatial and time criteria. Fig. 9 shows the verification and validation of the performance guarantee for the overall mission success, $P\left(r_{1} \leq R_{\max }, r_{2} \leq R_{\max }, t \leq T_{\max }\right)$, the probability that

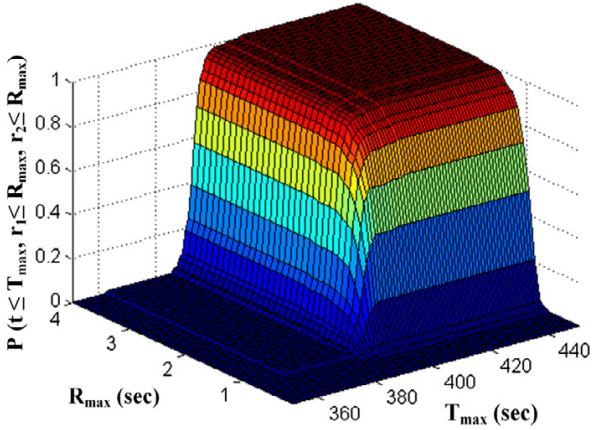

(a) Experimental validation.

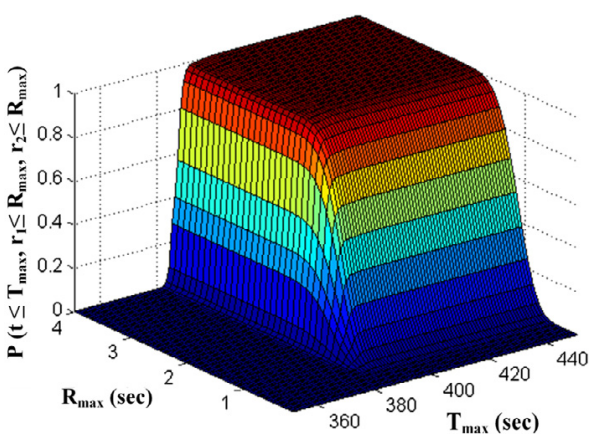

(b) VIPARS verification.

Fig. 9. Validation (a) and verification (b) of overall mission success $P\left(r_{1} \leq\right.$ $\left.R_{\max }, r_{2} \leq R_{\max }, t \leq T_{\max }\right)$

the bounding overwatch mission is completed under the time limit $T_{\max }$ and both robots are within $R_{\max }$ radius of their respective goal position. The effect of different combinations of performance criteria values is further examined in Figs. 10-11. Fig. 10 shows the verification and validation of the time criterion, $P\left(t \leq T_{\max }\right)$, at various fixed values of the spatial criterion, $R_{\max }$. We observed that $R_{\max }$ in both high confidence regions (i.e., $R_{\max } \leq 0.5 \mathrm{~m}$ and $R_{\max } \geq 2.0 \mathrm{~m}$, Fig. 6 ) has no effect on $P\left(t \leq T_{\max }\right)$. However, $R_{\max }$ in the Uncertain region (e.g., $R_{\max }=0.8 \mathrm{~m}, 1.0 \mathrm{~m}, 1.2 \mathrm{~m}$ ) has significant impact on $P\left(t \leq T_{\max }\right)$. Specifically, $P\left(t \leq T_{\max }\right)$ plateaus at different probability values for different $R_{\max }$ 's in the Uncertain region. For instance, for $R_{\max }$ of $1.2 \mathrm{~m}, P\left(t \leq T_{\max }\right)$ plateaus at 0.5228 , which is the value of $P\left(r_{1} \leq 1.2, r_{2} \leq 1.2\right)$ for the spatial criterion in Fig. 7.

There is a significant discrepancy between verification and validation of $P\left(t \leq T_{\max }\right)$ when $R_{\max }$ 's are in the Uncertain region ( $\max 400 \mathrm{~mm})$. Similar observations are made in Fig. 11 for $P\left(r_{1} \leq\right.$ $\left.R_{\max }, r_{2} \leq R_{\max }\right)$ at various values of the time criterion, $T_{\max }$. These observations reinforced our view that performance criteria within the Uncertain region should be avoided, or be moved into the High Confidence (Successful) region by modifying mission parameters such as modifying the mission velocity limits or use different robots or sensors. For this paper, no attempt was made to manually or automatically modify mission parameters to improve mission performance based on verification results; the focus here was on the initial comparison of verification and validation.

\section{Verifying missions with localization}

To assess the effectiveness of verification in providing performance guarantees for probabilistic robot behaviors, we analyze two waypoint missions where a robot is tasked to navigate through a series of waypoints toward a goal with behaviors that are based on probabilistic localization algorithms. VIPARS is used to investigate 


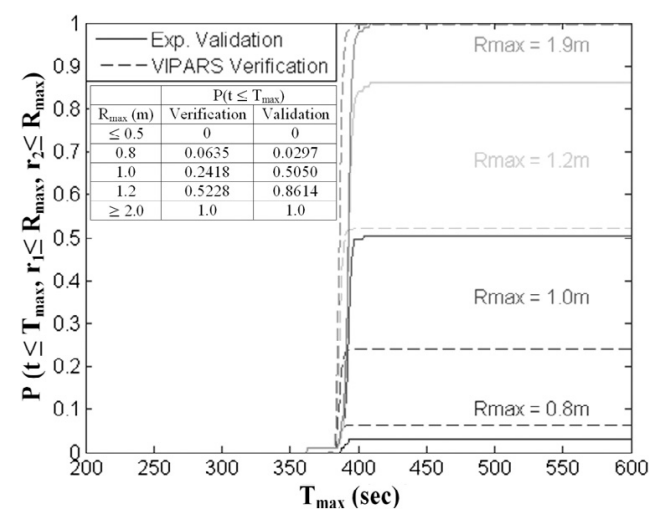

Fig. 10. Verification and validation of time criterion $P\left(t \leq T_{\max }\right)$ at various $R_{\max }$.

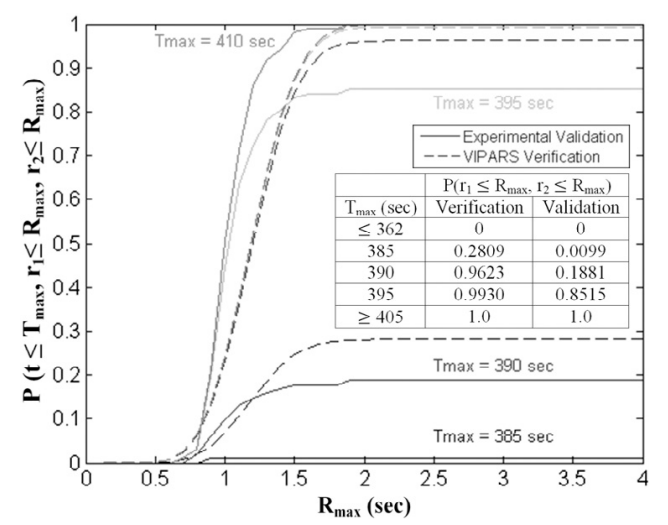

Fig. 11. Verification and validation of spatial criterion $P\left(r_{1} \leq R_{\max }, r_{2} \leq R_{\max }\right)$ at various $T_{\max }$.

two approaches to modeling localization and the results compared to experimental validation.

\subsection{Localization Missions-A and B}

Both missions proceed with a robot starting at $(2,2)$ in Fig. 12 and following a series of waypoints to the goal locations at (11.7, $12.5)$ and $(1.0,7.3)$ respectively for Mission- $A$ and Mission- $B$ (respectively). The behavior of the robot for Mission-B (Fig. 12(a)) is shown in Fig. 13, which was created in MissionLab in the form of a behavioral FSA. The robot FSA consists of a series of GoToGuarded and Spin behaviors, whose transitions are prompted by AtGoal and HasTurned triggers. The behavioral FSA for Mission-A is like the one shown in Fig. 13, and is omitted for brevity.

The perceptual schemas of MoveToGuarded and AvoidObstacles, two of the constituent primitive behaviors of the high-level GoToGuarded behavior, are augmented with a SLAM-based spatial map [7]. The MoveToGuarded primitive behavior drives the robot to a specified goal.

Instead of using odometry for localization, the perceptual schema of MoveToGuarded is replaced by an Adaptive Monte Carlo Localization (AMCL) algorithm [31]. This probabilistic localization algorithm takes the robot odometry and an a-priori acquired map as inputs, and outputs an estimated pose of the robot along with a covariance matrix representing the uncertainty of the estimated pose. Furthermore, the AvoidObstacles behavior uses the spatial map, instead of using direct sensory reading from the laser scanner, to generate repulsion vectors. The perceptual schema of the AvoidObstacles is modified to turn the spatial map into pseudo laser scans of the environment through beam tracing within the occupancy map. As a result, the GoToGuarded behavior utilizes perceptual information (i.e., robot pose and obstacles) generated by probabilistic algorithms to generate motor response while navigating through the waypoints.

The performance criteria for both missions are similar:

Success $=\left(r \leq R_{\max }\right)$ and $\left(t \leq T_{\max }\right)$

where $R_{\max }$ is maximum radius of spatial deviation allowed from the goal and $T_{\max }$ is the maximum allowable mission completion time, and where $r$ is the robot's relative distance to its goal location and $t$ is the time the robot to finish a mission.

\subsection{Localization mission system process}

The input to VIPARS is the system process composed of the behavior FSA from MissionLab converted to PARS and combined with the PARS models for the robot, sensors and environment. The system process Sys for the localization mission is shown in Eq. (16).

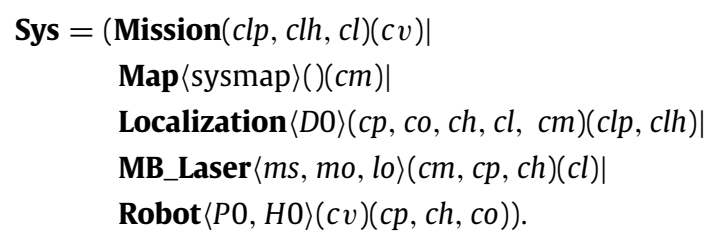

The Mission process is the translation of the waypoint mission and is fundamentally similar to all prior waypoint missions we have verified and validated. It has inputs $\mathrm{clp}$ (position), $\mathrm{clh}$ (heading) and $\underline{c l}$ (laser readings); and output $c v$ (velocity). Robot is the environment model, capturing the motion and odometry error and interactions with obstacles, as before. $\mathrm{PO}, \mathrm{HO}$ are initial position and heading, inputs $c v$ (velocity) and outputs $c p$, $c h$ (odometry position and heading) and co (real position distribution, i.e., without sensing noise-only used for performance estimation and highlevel localization model).

However, there are three new processes: In the behavior-based localization approach [7], the obstacle avoidance sensor gets its information from the map, rather than directly from measuring sensory input. Map makes mapping information (from the a-priori generated sysmap) available on its output $\mathrm{cm}$; MB_Laser uses the map to generate map-based laser data on its output $c l$. Localization implements a localization method using the map $\mathrm{cm}$, laser $\mathrm{cl}$, and robot $c p, c o$, $c h$ inputs. $D 0$ is the initial position uncertainty. The output of Localization, $c l p$, is the localized position (and heading $\mathrm{clh}$ ) used by the Mission process.

\subsection{Map representation}

A key difference between this localization mission and our prior missions including bounding overwatch is the map and the role it plays in the obstacle avoidance behavior and in localization. The Map process in Eq. (16) contains a map data structure. Recall that variables in a PARS process definition can be random variables represented as colored mixtures of Gaussians distributions (CMG).

Map information - the locations and geometry of obstacles, walls and other physical aspects of the mission environment - can be directly represented using this model. The interactions of the map with the robot and map-based sensor is analyzed in VIPARS by measuring the overlap between random variable distributions, Eq. (12). The advantage of this approach to representing physical geometry is that there is no restriction on the spatial location or extent of obstacles, and finer precision of modeling can be obtained at the cost of adding more mixture members (Fig. 14).

Definition. An indexed mixture of Gaussians is a mixture of Gaussians distribution $a \sim M G(C M)$ together with an index set $I$. The mixture is restricted as follows: 

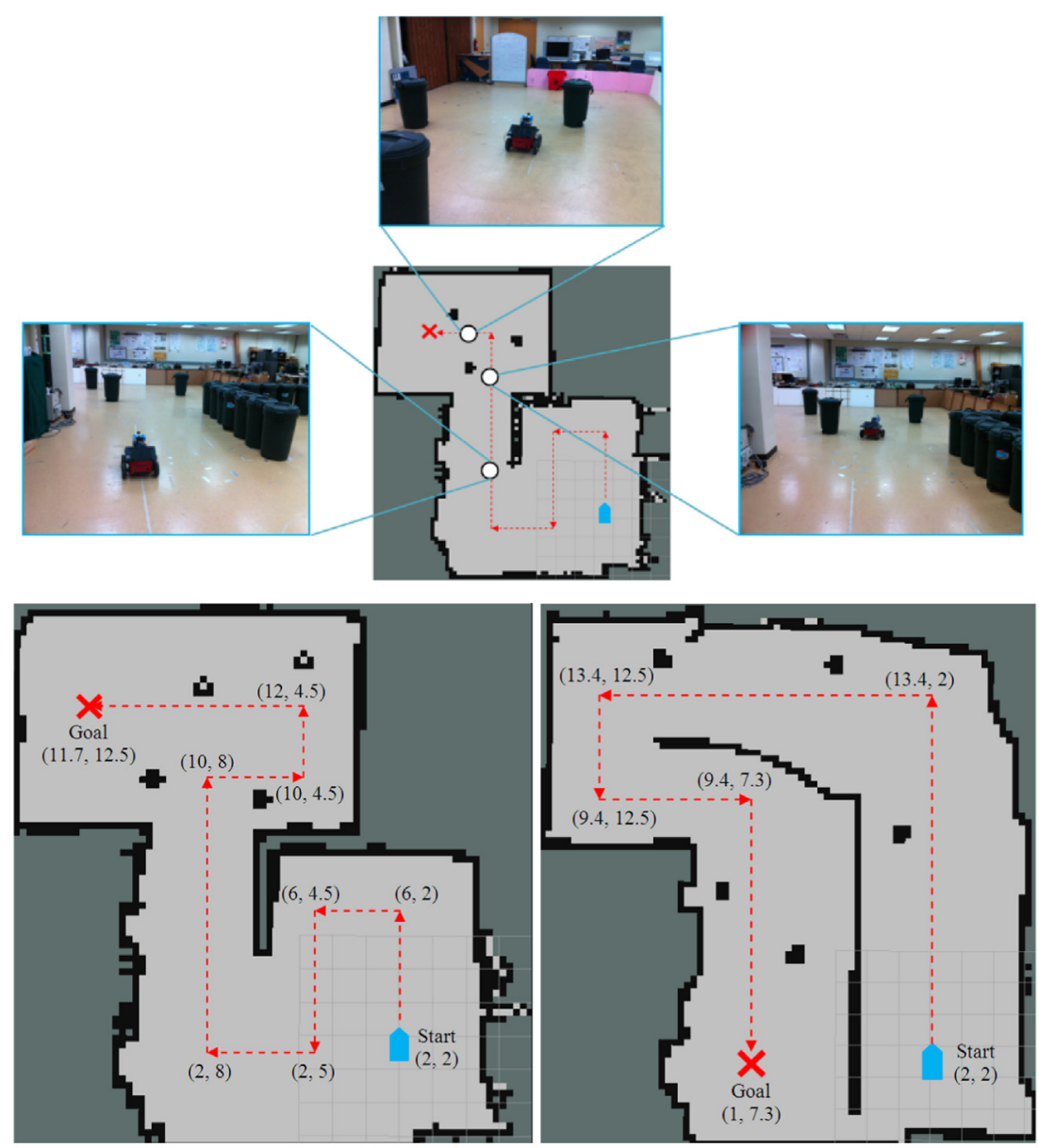

(a)Waypoints Mission A.

(b) Waypoints Mission B.

Fig. 12. (top) Example operating environment images for localization missions; (bottom a, b) two waypoint missions for verification and validation.

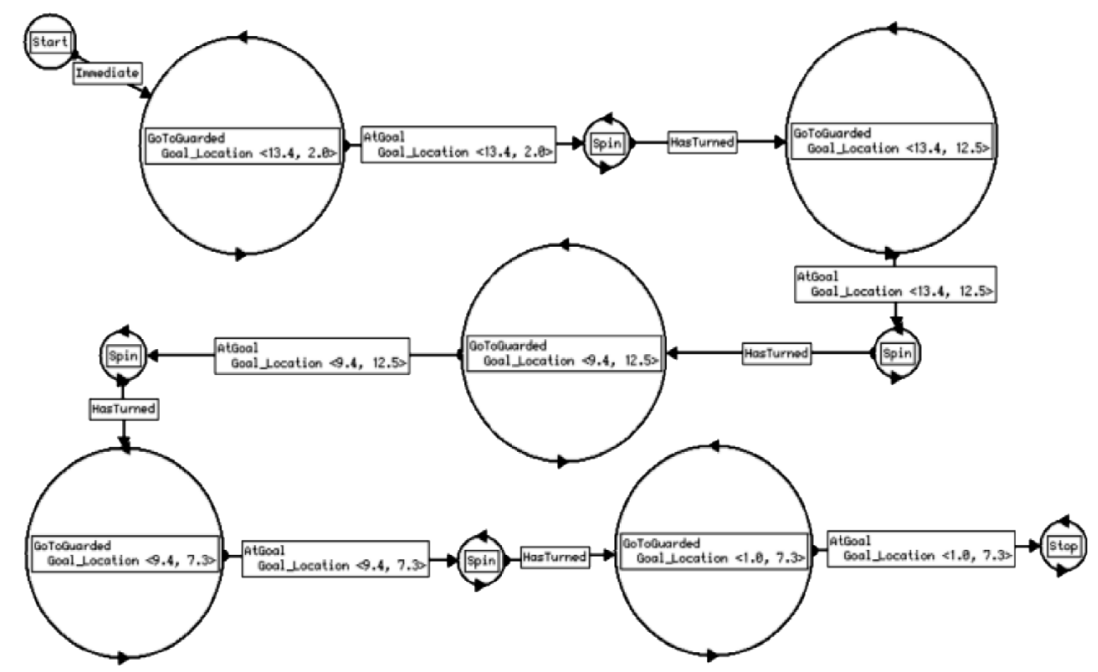

Fig. 13. Behavioral FSA for Mission-B.

- $a[x] \equiv a_{i}$ where $\mu\left(a_{i}\right)=x \in I, i \in 1 \ldots m$.

- $\mu\left(a_{i}\right) \in I$, for all $i \in 1 \ldots m$; $a$ only contains members indexed by $\underline{I}$.
- For any $x \in I,|\{a[x]\}| \leq 1$; $a$ has at most one member for each index.

A map is defined as an indexed bivariate mixture of Gaussians where $I=[0 \ldots X] \times[0 \ldots Y]$ and where each member is a 


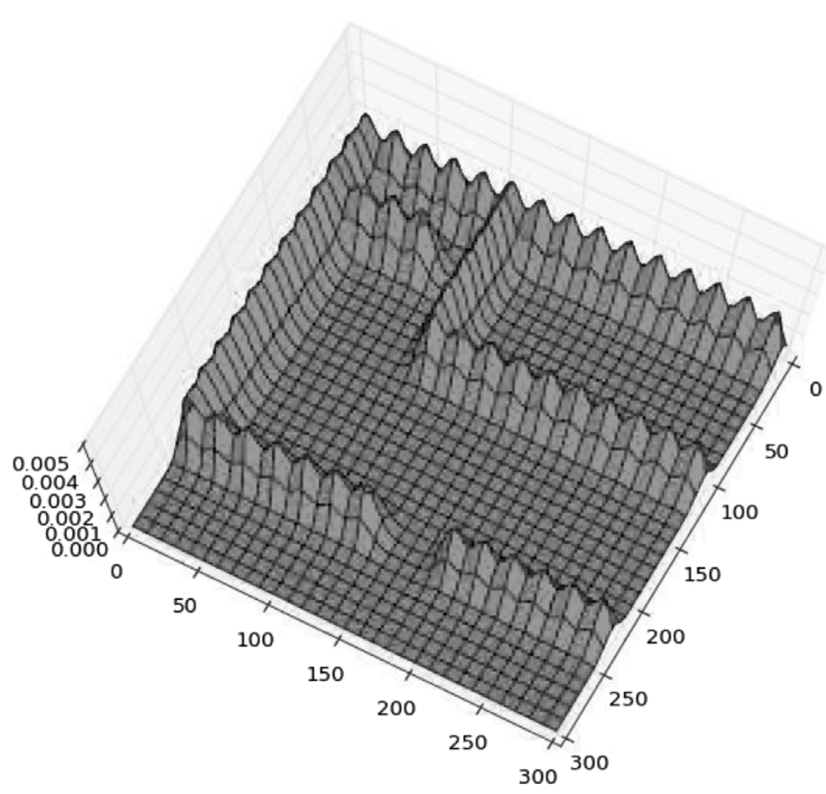

Fig. 14. Example VIPARS map representation.

Gaussian kernel with covariance $\Sigma[x, y]=\sigma_{m}^{2} I$, and where $\sigma_{m}$ represents the map resolution. This corresponds somewhat intuitively with an occupancy grid representation [32], where $w[x, y]$ is related to probability of occupancy for the location $(x, y)$.

During verification, the location random variable (the connection $c p$ in Eq. (16)) represents the location of the robot for all possible executions. It is relevant to compare this with the representation of robot location in a localization algorithm: the representation there may also be a random variable, but the interpretation is different. In any single execution, the robot can really only be at a single physical location; the localization distribution is an estimate of this. In verification, the objective is not to find the single most likely location, but to propagate the effects of being at all locations. Rather than using a ray trace algorithm to determine how each location is supported by sensor readings and refining the position estimate based on that, the ray trace algorithm is used by the MB_Laser process to gather all possible sensor readings that can arise due to the robot location distribution.

\subsection{Modeling localization}

The first approach involves modeling localization at a high level: modeling not the actual collection of sensory data that produces improved position estimates, but just position estimates that improve with time according to some parameterization. This has the advantage that different localization algorithms can be 'modeled' in verification by just changing the parameterization, not requiring as many hours of expert effort as implementing a new localization algorithm directly in the formal framework. It has the disadvantage that it decouples the localization from predicted sensor measurements, and may miss the effect of measurements that greatly improve or degrade the localization estimate.

The second approach involves the incorporation of existing localization code directly into the VIPARS verification algorithm. Localization code, like any program, when executed, will yield one possible trace of a robot mission, whereas VIPARS needs to use that code to probabilistically reason about all executions that are possible given the a-priori environment model information. Our approach considers the embedded code to be capable of transforming a sample from a PARS random variable, and we define a framework for sampling and reconstructing variable distributions. This approach has the advantage of verifying the actual preexisting, localization code that will get executed by the robot at run-time for the mission. It has the disadvantage of potentially lengthening verification times, since multiple samples need to be evaluated for a representative result.

\subsubsection{High-level model approach}

Localization starts with the odometry estimate of position at time step $t, q(t) \sim M G$. Through comparisons of sensory returns and the map, it refines the odometry estimate, bringing it closer to the actual position of the robot at time $t, p(t) \sim M G$. At any time, therefore the localization position is some combination of the odometry and the actual position:

$\ell(t)=(1-k(t)) p(t)+k(t) q(t)$

where $k(t) \in[0,1]$ is a time varying gain with $k\left(t_{0}\right)=1.0$, forcing localization to start with just the odometry estimate. The improvement of localization with time is modeled by a monotonicdecreasing dynamics for $k$ :

$k(t+\Delta t)=t_{c} k(t)$.

For time constant, $t_{c} \in[0,1]$, determined from calibration measurements of the localization algorithm to be verified.

\subsubsection{Sampling approach}

Consider that a preexisting $\mathrm{C}++$ program we want to include in a mission is $\mathbf{P}$. A PARS process wrapper for $\mathbf{P}$ is built, so the code behaves like a 'black box' process $\mathbf{P}\langle x\rangle\langle y\rangle$. Then, like every PARS process, it has an associated flow function $f_{P}(x)=(y)$. However, when $\mathbf{P}$ is called, it will map one input value $x$ to an output, $y$; only one possible execution of $\mathbf{P}$, whereas verification has to check all possible executions. So, this approach to embedding $\mathbf{P}$ does not work, but, embedded code can only be called in this way.

Our approach is to define an extension to the flow function $f_{P}$ from the process/program $\mathbf{P}$ : the mixture extended flow function $F_{P}$ takes a random variable $x$ as input and produces a random variable $\mathbf{y}$ as output. It samples the input distribution $x$ and calls $f_{P}$ on the samples, and reconstructs the output distribution mixture $p(y \mid x)=F_{P}(\boldsymbol{x})$ from the result.

Definition. Let $f_{P}(x)=y$ be the flow-function for the code to be embedded in verification, defined only by executing that code. Let $\boldsymbol{x}, \boldsymbol{y} \sim M G(C M)$ be random variables over the type of the variables $x, y$ which we denote $T$. The mixture extended flow function (MEF) $F_{P}$ is defined as follows.

- $f_{P}: T \rightarrow T$, where $y=f_{P}(x)$, for $x, y \in T$,

- $F_{P}$ : MG $\rightarrow$ MG, where $\mathbf{y}=F_{P}(\boldsymbol{x})$, for $\boldsymbol{x}, \boldsymbol{y} \in$ MG (where MG is the set of all $M G$ ), and

- where we define $\mathbf{y}=\mathbf{x}$

- except $\boldsymbol{\mu}\left(y_{i}\right)=f_{P}\left(\boldsymbol{\mu}\left(x_{i}\right)\right)$ for all $x_{i}$ in $\boldsymbol{x}$, and

- where $\boldsymbol{\sigma}\left(y_{i}\right)$ is calculated as follows:

$$
\begin{array}{ll}
\circ & \boldsymbol{\mu}_{j}^{\prime}=f_{P}\left(s_{i}\right) \text { for } s_{i} \text { a sample of the input } x_{i} \\
\circ & \sigma\left(y_{i}\right)=\sum_{j=1}^{k} N\left(s_{j} ; \mu\left(x_{i}\right), \sigma\left(x_{i}\right)\right)\left(\left(\mu_{j}^{\prime}-\mu\left(y_{i}\right)\right)^{2}\right) .
\end{array}
$$

The MEF preserves the number of members $(|\mathbf{y}|=|\mathbf{x}|)$. Each mean is transformed directly $\boldsymbol{\mu}\left(y_{i}\right)=f_{\mathrm{P}}\left(\boldsymbol{\mu}\left(x_{i}\right)\right)$, requiring multiple executions of the embedded code. Finally, each variance is calculated by carrying out further sample executions for each member $\boldsymbol{\mu}_{j}^{\prime}=f_{P}\left(s_{i}\right)$. 


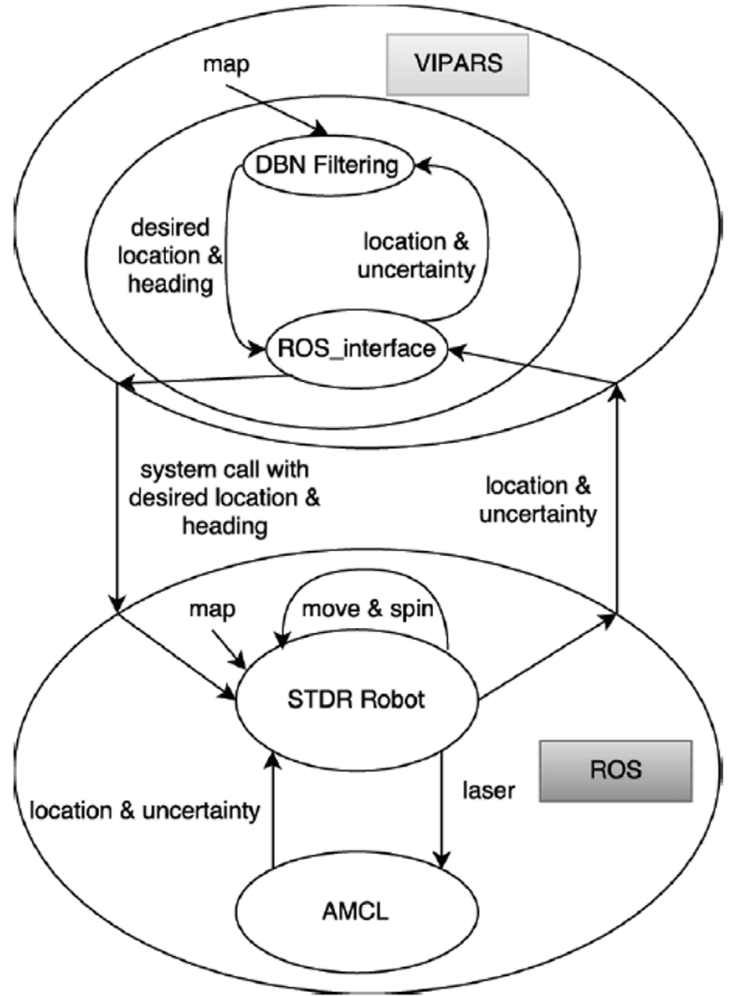

Fig. 15. VIPARS-ROS architecture.

\subsection{Embedding ROS AMCL localization}

The localization algorithm used in this paper was Adaptive Monte Carlo Sampling (AMCL) [33] as implemented in ROS. In the sampling approach, the DBN filtering engine of VIPARS issued requests to a ROS-based AMCL server to evaluate the MEF function for Localization. The interaction is shown in Fig. 15: Whenever the flow function for the Localization process needed to be evaluated on a position random variable, the position variable was sent from the DBN filtering engine (Top, Fig. 15) via a pipe to a concurrently running ROS system (Bottom, Fig. 15). The STDR simulator node was instructed to move and rotate ("move and spin" in Fig. 15) the robot to the appropriate position, and localization data collected from the AMCL node. For simplicity, the MEF function was restricted to single member variables, and rather than calculating the variance by evaluating multiple samples, only the mean value was transformed and the variance calculated by convolving the mean with a zero-mean distribution $N\left(0, \sigma_{s}\right)$. This simplified the hysteresis issue with calling AMCL.

\subsection{Verification}

Both verification approaches were applied to both waypoint missions. For the high-level approach, Localization in Eq. (16) implemented Eqs. (17), (18) with the gain parameter $t_{c}=0.99$. This value was empirically determined from experimentation running ROS AMCL on a Pioneer 3-AT robot, carrying out a series of short waypoint missions.

The sample-based approach implemented the architecture of Fig. 15 using ROS Indigo. A third, odometry-only version of the mission was also run through VIPARS for comparing with both localization methods, and determining whether localization was really necessary for mission success. No additional validation was

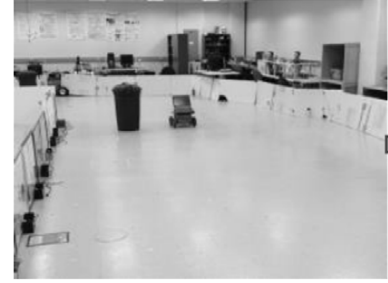

(a)Robot moving toward $1^{\text {st }}$ waypoint.

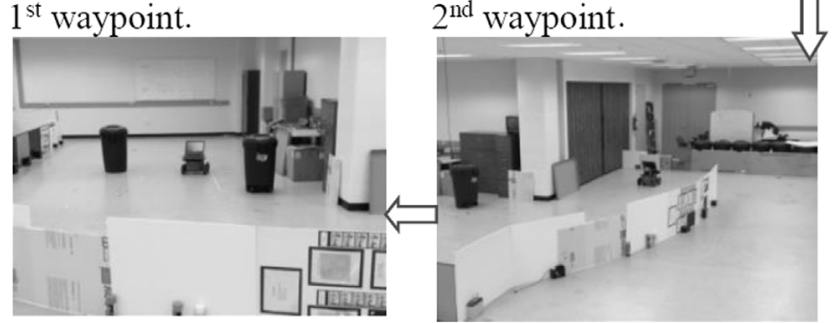

(d) Robot moving toward goal location.

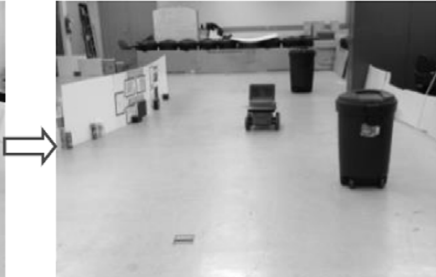

(b) Robot moving toward $2^{\text {nd }}$ waypoint. (c) Robot after turning a corner.

Fig. 16. Snapshots of validation for Mission-B.

done on the odometry-only version since that just replicates our prior work [1].

The results of carrying out verification using both approaches with both waypoint missions was a set of performance graphs showing the predicted performance of the missions with respect to the performance criteria.

\subsubsection{Validation}

The robot used for the experimental trials is the Pioneer 3$A T$, a four-wheeled skid-steered mobile robot. The robot is also equipped with a forward-facing SICK laser scanner. The complete validation experiment consists of 50 trial runs for each waypoint mission respectively, which resulted in a total of 100 trial runs. Snapshots of the waypoint mission B are shown in Fig. 16. For each trial, mission completion time and relative distance to goal on completion were measured.

\subsubsection{Comparison of verification and validation results}

Fig. 17 shows the validation results of the performance guarantees for the two waypoint missions. These results are obtained with the sampling-based model of probabilistic localization. Fig. 17(a) and (c) show the $\mathrm{V} \& \mathrm{~V}$ results for the spatial criteria $P\left(r \leq R_{\max }\right)$, the probability that the robot arrives within $R_{\max }$ radius of its goal location. Fig. 17(b) and (d) show the comparisons for the time criteria $P\left(t \leq T_{\max }\right)$, the probability that the waypoint mission is completed under the time limit, $T_{\max }$. The results illustrate that the VIPARS verification of performance guarantees are consistent with the outcomes from experimental validation. The V\&V results can be divided into three regions for further interpretation as before: High Confidence (Unsuccessful), Uncertain, and High Confidence (Successful) region. Consequently, the mission operator's decision for robot deployment can be based on which region of the mission criteria fall into. For instance, if the specified performance criterion falls within the Unsuccessful region (e.g., $R_{\max }=0.5 \mathrm{~m}$ ), the operator can either abort the mission or modify mission parameters and reevaluate. The overall mission success, Eq. (15), is defined in terms of both spatial and time criteria. Thus, we examined further in Figs. 18 and 19 the effects of various combinations of spatial and time criteria $\left(R_{\max }\right.$ and $\left.T_{\max }\right)$ on the mission success and verification error. The results can also be used to answer queries regarding the performance guarantee for a specific combination of $T_{\max }$ and 


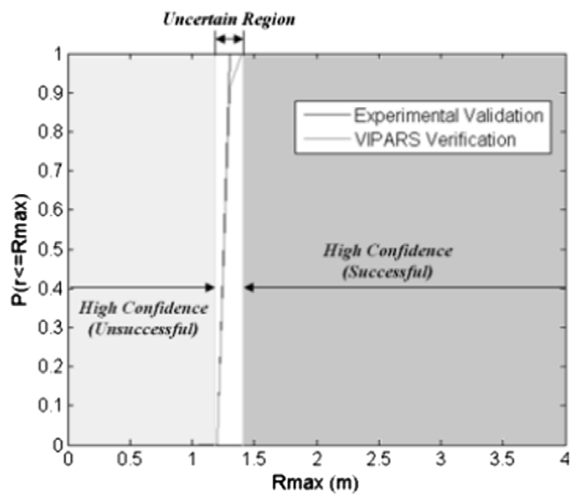

(a) Mission A spatial criterion $P\left(r \leq R_{\max }\right)$.

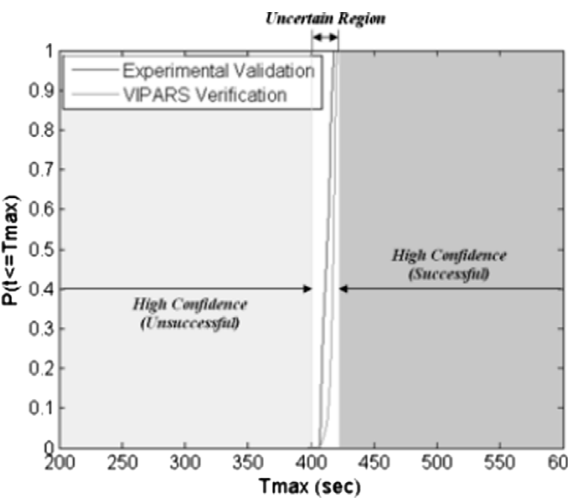

(b) Mission A time criterion $P\left(t \leq T_{\max }\right)$.

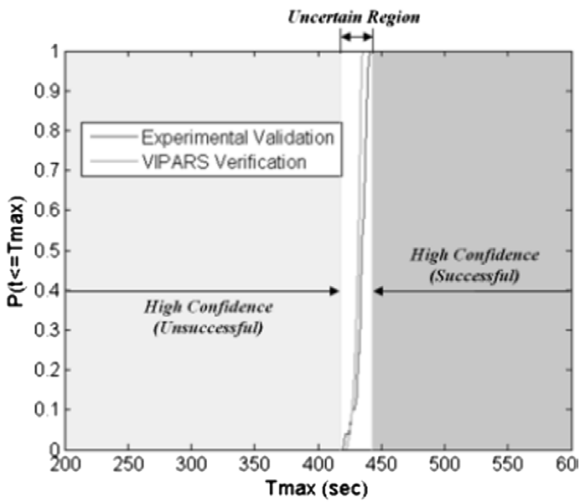

(d) Mission B time criterion $P\left(t \leq T_{\max }\right)$.

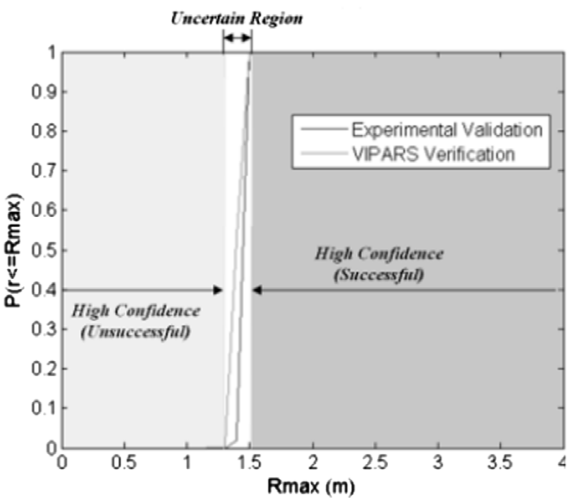

(c) Mission B spatial criterion $P\left(r \leq R_{\max }\right)$.

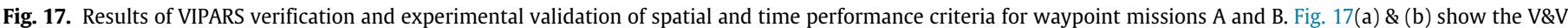

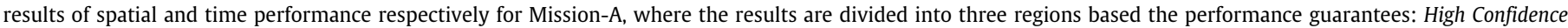
(Unsuccessful), Uncertain, and High Confidence (Successful). Fig. 17(c) and (d) show the V\&V results for mission-B.

$R_{\max }$. Fig. 18 shows the effects of the time criterion $T_{\max }$ on the $\mathrm{V} \& \mathrm{~V}$ results of the spatial criterion $P\left(r \leq R_{\max }\right)$ for Mission $A$. While the $T_{\max }$ 's in both of its high confidence regions (Fig. 17(b)) have no effect on the verification error for $P\left(r \leq R_{\max }\right), T_{\max }$ 's that are in the Uncertain region (e.g., $T_{\max }=415 \mathrm{~s}$ ) incur significant verification errors. For instance, for $T_{\max }=415 \mathrm{~s}$, VIPARS predicted a success probability of 0.18 , while the robot was actually successful $76 \%$ of the time in experimental trials. Fig. 19 shows the effects of the spatial criterion $R_{\max }$ on the V\&V results of the time criterion $P\left(t \leq T_{\max }\right)$. While similar observations can be made here as in Fig. 18 , in this case, $R_{\max }$ 's have much less impact on the verification error of $P\left(t \leq T_{\max }\right)$ due to VIPARS's accuracy in predicting the spatial performance of mission even in the uncertain region (as shown in Fig. 17(a)). Nonetheless, our conclusion is that missions with performance criteria in the Uncertain regions should generally be avoided.

Lastly, we have also examined the different verification results of VIPARS based on how the probabilistic localization mechanism is modeled: sampling-based and high-level model-based. These results are also compared in Mission A to the verification result for the case when only odometry information is used for localization. These verification results are shown in Figs. 20-21 along with the validation result for Mission-A. While the verification results for different localization modeling approaches are comparable for the time criterion (Fig. 20), the performance based on the samplingbased model is more closely aligned with the validation result for both spatial and time criteria. If only high-probability results are of interest, then the simpler and faster, model-based localization produces acceptable results.

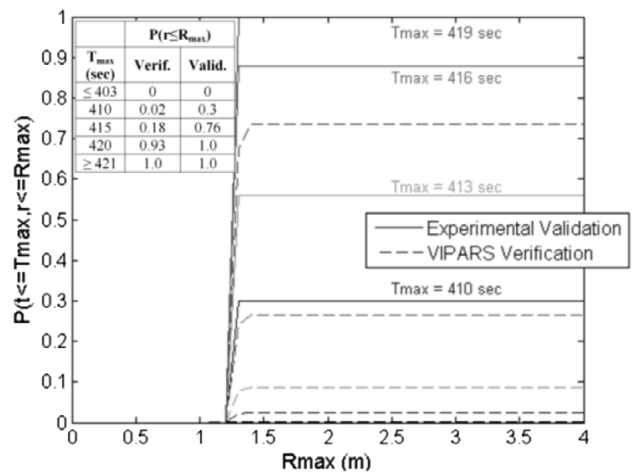

Fig. 18. $V \& V$ of spatial criterion at various $T_{\max }$ for Mission $A$.

The odometry-only Mission B was 100\% unsuccessful during verification due to collisions. However, with the final waypoints moved just $15 \mathrm{~cm}$, the odometry-only mission finishes successfully. Because a small modification enables the odometry-only mission to be potentially successful, it is also clear that localization is not always required for mission success. A contribution of our approach is that it is now possible to answer whether localization is of mission benefit using the performance graphs below in conjunction with the specific performance values of $R_{\max }$ and $T_{\max }$. Being able to omit software modules (such as localization) can yield lighter and faster mission code. 


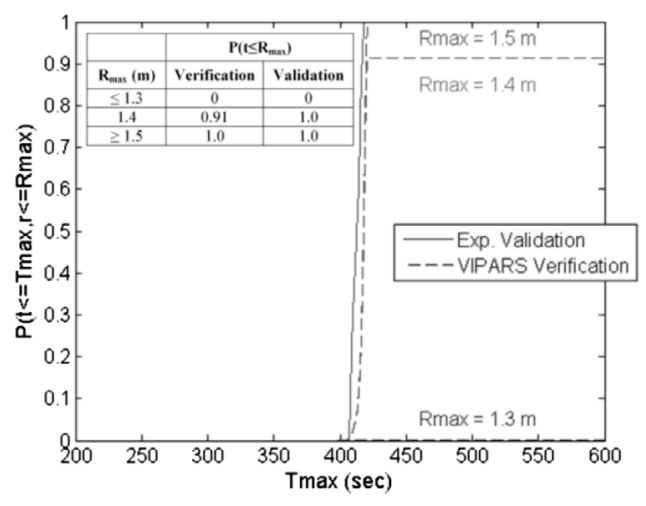

Fig. 19. $\mathrm{V} \& \mathrm{~V}$ of time criterion at various $R_{\max }$ for Mission $A$.

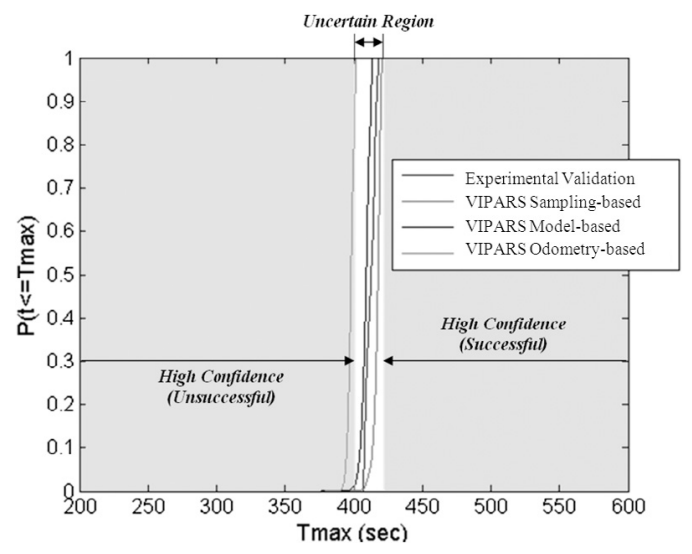

Fig. 20. V\&V of time criterion and models of localization.

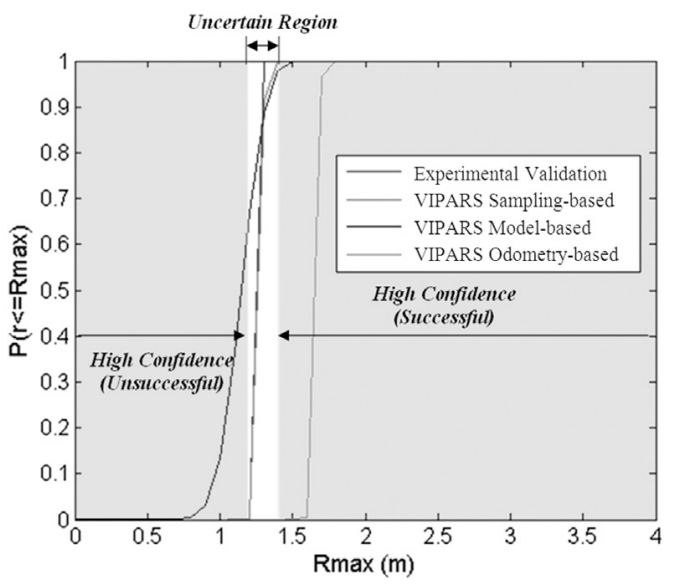

Fig. 21. V\&V of spatial criterion and models of localization.

\section{Conclusions}

If teams of autonomous robots are to undertake critical missions such as C-WMD missions, then it is vital to be able to establish performance guarantees for them. This paper addresses the challenge of verifying mission software for autonomous robots that will operate in partially known environments. The approach taken in this paper, and its predecessor [1], differs from common approaches to robotic software verification in two important ways: it emphasizes the roles of a separate but communicating environment model, and it eschews an explicit exploration of the state space of the combined mission software and environment model for reasons of avoiding state-space explosion. This paper significantly expands [1], which addressed uncertainty in robot motion and sensing, by addressing uncertain geometry in the environment model.

Two classes of mission were investigated: a mission where a team of two robots executes a coordinated set of motions during which they may encounter obstacles whose existence and location is uncertainly known in advance, and a robot mission in which the robot navigates a series of waypoints leveraging probabilistic localization. Approaches to representing and analyzing both mission classes were presented. In addition, separately collected experimental validation results were presented for both classes of mission and were compared to the results from verification.

The comparison of experimental validation and the output of the verification software show the effectiveness of the verification framework in providing performance guarantees for multi-robot missions operating in an uncertain environment. Some of the noted discrepancies between verification and validation may be due to calibration inaccuracies but also the precision limitation from pruning CMG variables.

The colored mixtures developed here may have wider applications. Algorithms that selectively modify mixture members (e.g., image background update [34], in addition to those discussed here) can thus easily propagate subpopulations of one or more members identified for later processing. With respect to complexity and scaling: The computation of $s(t) \sim C M G$ just increases linearly with each additional obstacle (and robot), but each robot must evaluate its own copy. The number of members increases exponentially with each filtering step. In this paper, they were pruned on weight to a maximum of 10 .

The multiple robot synchronization in this paper involved direct and indirect (i.e., through the environment model) interactions. The robots directly exchanged synchronization messages as they completed each mission bound. Although no communication latency or error was modeled here, it is a straightforward extension to model for example, WiFi limitations. The indirect interaction was limited to the robots being able to view each other as obstacles. While this generalizes easily to any number of robots (the principal complexity is just evaluating Eq. (12)) it does not model contact interaction between the robots such as one pushing the other or both physically collaborating on a task.

The paper also addressed verification of missions with probabilistic localization. Two approaches to modeling localization were presented and evaluated: a high-level approach in which only position estimate improvement is modeled, and a sample-based approach, in which the run-time localization code is embedded in verification. Extensive experimental validation is reported for two different waypoint missions using localization. The sample-based approach yields the more accurate estimate, even for the sampling simplification made in this paper. While there is support for the intuition that localization is an asset to mission performance (100\% failure of the non-odometry mission; Mission B of Section 5.6); a minor modification of $15 \mathrm{~cm}$ will allow the mission to be verified successful, indicating that the need for localization is missionspecific.

A verification tool is only as effective as its usability [35]. Therefore, a key future direction for this work is the challenge of presenting verification results to the mission designer in an intuitive and effective way. A second thrust of continuing work is the extension and evaluation of this approach for missions that include a human in the loop element.

\section{References}

[1] D. Lyons, R. Arkin, S. Jiang, T.-L. Liu, P. Nirmal, Performance verification for behavior-based robot missions, IEEE Trans. Robot. 31 (3) (2015). 
[2] D. MacKenzie, R. Arkin, R. Cameron, Multiagent mission specification and execution, Auton. Robots 4 (1) (1997) 29-52.

[3] R. Jhala, R. Majumdar, Software model checking, ACM Comput. Surv. 41 (4) (2009).

[4] P. Trojanek, K. Eder, Verification and testing of mobile robot navigation algorithms, in: IEEE/RSJ Int. Conf on Intelligent Robots and Systems, IROS, Chicago, 2014.

[5] D. Walter, H. Taubig, C. Luth, Experiences in applying formal verification in robotics, in: 29th International Conference on Computer Safety, Reliability and Security, Vienna Austria, 2010.

[6] L. Kiekbusch, C. Armbrust, K. Berns, Formal verification of behavior networks including sensor failures, Robot. Auton. Syst. 74 (2015) 331-339.

[7] S. Jiang, R. Arkin, SLAM-based spatial memory for behavior-based robots, in: 11th IFAC Symposium on Robot Control, SYROCO, Salvador, Brazil, 2015.

[8] L. DeMoura, N. Bjorner, Satisfiability modulo theories: Introduction and applications, CACM 54 (9) (2012) 54-67.

[9] A. Cowley, C. Taylor, Towards language-based verification of robot behaviors, in: IEEE/RSJ Int. Conference on Int. Robots and Systems, IROS, 2011.

[10] T. Ropertz, R. Berns, Verification of behavior-based networks-using satisfiability modulo theories, in: ISR/Robotik 2014; 41st Int. Symposium on Robotics, 2014.

[11] M. Kim, K.-C. Kang, H. Lee, Formal verification of robot movements - a case study on home service robot SHR100, in: IEEE Int. Conf. Rob. \& Aut., 2005.

[12] M. Webster, C. Dixon, M. Fischer, M. Salem, J. Saunders, K.-L. Koay, K. Dautenhahn, Formal verification of an autonomous personal robotic assistant, in: AAAI 2014 Symposioum Modeling in Human-machine Systems: Challenges for Formal verification, Stanford CA, 2014.

[13] M. Fisher, L. Dennis, M. Webster, Verifying autonomous systems, CACM 56 (9) (2013) 84-93.

[14] M. Guo, K. Johansson, D. Dimarogonas, Revising motion planning under temporal logic specifications in partially known workspaces, in: IEEE. Int. Conf. Rob. \& Aut., 2013.

[15] S. Sarid, B. Xu, H. Kress-Gazit, Guaranteeing high level behaviors while exploring partially known maps, in: Rob. Sc. \& Sys., Vol. 2013, 2008.

[16] M. Proetzsch, K. Berns, T. Schuele, K. Schneider, Formal verification of safety behaviours of the outdoor robot ravon, in: 4 th Int. Conf. on Informatics, Aut. and Control, Dortmund, Germany, 2007.

[17] A. Zaks, R. Joshi, Verifying Multi-threaded C programs with SPIN, in: 15th International SPIN Workshop, Los Angeles CA, 2008.

[18] M. O'Brien, R. Arkin, D. Harrington, D. Lyons, S. Jiang, Automatic verification of autonomous robot missions, in: 4th Int. Conf. on Simulation, Modelling and Prog. for Aut. Robots, Bergamo, Italy, 2014.

[19] H. Kress-Gazit, G. Pappas, Automatic synthesis of robot controllers for tasks with locative prepositions, in: IEEE Int. Conf. on Rob. \& Aut., Anchorage, Alaska, 2010.

[20] S. Bensalem, K. Havelund, A. Orlandini, Verification and validation meet planning and scheduling, Int. J. Softw. Tools Technol. Transfer 16 (1) (2014) 1-12.

[21] D. MacKenzie, R. Arkin, Evaluating the usability of robot programming toolsets, Int. J. Robot. Res. 4 (7) (1998) 381-401.

[22] R.C. Arkin, Behavior-Based Robots, MIT Press, Cambridge MA, 1998.

[23] A. Roscoe, The Theory and Practice of Concurrency, Prentice-Hall, 1997.

[24] T. Bolognesi, E. Brinksma, Introduction to the ISO specification language LOTOS, Comput. Netw. ISDN Syst. 14 (1) (1987) 25-59.

[25] M. Steenstrup, M. Arbib, E. Manes, Port automata and the algebra of concurrent processes, JCSS 27 (1) (1983) 29-50.

[26] D. Lyons, R. Arkin, T.-L. Liu, S. Jiang, P. Nirmal, Verifying performance for autonomous robot missions with uncertainty, in: IFAC Intelligent Vehicle Symposium, Gold Coast Australia, 2013.

[27] S. Russel, P. Norvig, Artificial Intelligence, Prentice-Hall, 2010.

[28] D. Lyons, R. Arkin, S. Jiang, D. Harrington, F. Tang, P. Tang, Probabilistic verification of multi-robot missions in uncertain environments, in: IEEE Int. Conf. on Tools with AI, Vietro sul Mare, Italy, 2015.

[29] R. Szczerba, B. Collier, Bounding overwatch operations for robotic and semirobotic ground vehicles, in: SPIE Aerospace Conference on Guidance and Navigation, 1998

[30] A. Bhattacharyya, On a measure of divergence between two statistical populations defined by their probability distributions, Bull. Calcutta Math. Soc. 35 (1943) 99-109

[31] F. Dellaert, D. Fox, W. Burgard, S. Thrun, Monte Carlo localization for mobile robots, in: IEEE Int. Conf. on Rob. \& Aut., Detroit, 1999.

[32] A. Elfes, Using occupancy grids for mobile robot perception and navigation, Computer (1989) 48-57.

[33] D. Fox, KLD-Sampling: Adaptive particle filters, in: Neural Information Processing Systems, Vol. 14, NIPS, Vancouver Canada, 2001

[34] C. Stauffer, W. Grimson, Adaptive background mixture models, in: CVPR, 1999.

[35] M. O'Brien, R. Arkin, An analysis of displays for probabilistic robotic mission verification results, in: 7th International Conference on Applied Human Factors and Ergonomics, Las Vegas NV, 2016.

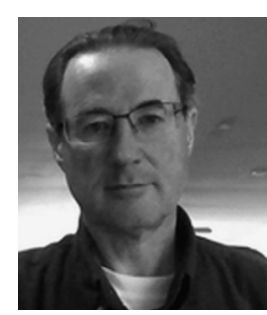

D. M. Lyons is a Professor of Computer Science at Fordham University, and Director of Fordham's Robotics \& Computer Vision Laboratory. He has degrees in Math, Engineering, and Computer Science from Trinity College, University of Dublin, Ireland, and a Ph.D. in Computer Science from the University of Massachusetts. His research interests include formal approaches to plan and program analysis and robot team exploration strategies. Prior to joining Fordham, he was a senior researcher and department head at Philips Corporate Research, NY. He has served as Chair an IEEE Senior Member. of the IEEE RAS TC on Assembly and Task Planning and is

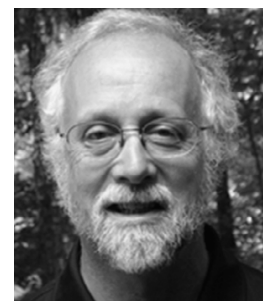

R. C. Arkin is Regents' Professor and Associate Dean in College of Computing at Georgia Tech. He served as STINT visiting Professor at KTH Stockholm, Sabbatical Chair at Sony IDL in Tokyo, and in the Robotics/AI Group at LAAS/CNRS in Toulouse. His research interests include behavior-based control and action-oriented perception, deliberative/reactive architectures, multiagent robotics, biorobotics, human-robot interaction, and robot ethics. He served on the Board of Governors of IEEE Society on Social Implications of Technology, IEEE Robotics and Automation AdCom, and as founding co-chair of IEEE RAS TC on Robot Ethics. He is a Distinguished Lecturer for the IEEE SSIT and an IEEE Fellow.

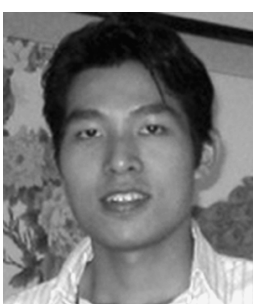

S. Jiang is a Robotics Ph.D. student in the Institute for Robotics and Intelligent Machines at Georgia Institute of Technology and a member of the Mobile Robot Laboratory. He received B.S. and M.S. degrees in Electrical Engineering from the University of Florida in 2009. His research interests include formal methods, human-robot teams, educational and assistive robotics.

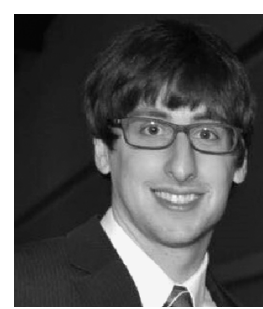

M. O'Brien is a Robotics Ph.D. student at Georgia Institute of Technology, currently working in the Mobile Robot Laboratory. He received his B.S. in Electrical Engineering from the University of Alabama in 2013. His research interests surround long-term autonomy and dynamic environments for mobile robots.

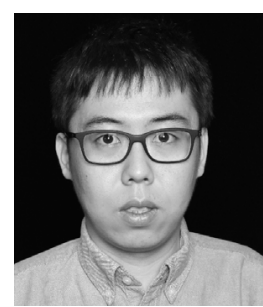

F. Tang is a Software Engineer. He received B.S. and M.S degrees in Information Security from Shanghai Jiao Tong University, China, and an M.S. degree in Computer Science from Fordham University, NY. His research interests are machine learning, robotics and computer vision.

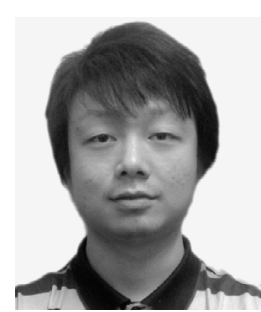

P. Tang work as a Data Engineer at DST Systems. He Obtained his bachelor's degree in Communications Engineering from Changsha University of Science and Technology and master's degree in Computer Science at Fordham University. His area of interests include artificial intelligence algorithms, machine learning and developing scalable big data products. 